Boletín de la Sociedad Geológica Mexicana

VOLUMEN 66, NÚM. 1, 2014, P. 145-164

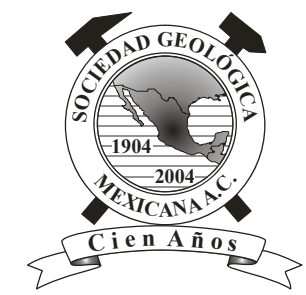

\title{
Una especie de delfín fósil (Cetacea; Delphinoidea; Kentriodontoidae) del Mioceno Medio de Baja California
}

\author{
Fernando M. Salinas-Márquez ${ }^{1}$, Lawrence G. Barnes ${ }^{2}$, Juan G. Flores-Trujillo ${ }^{3}$, Francisco J. \\ Aranda-Manteca ${ }^{1, *}$ \\ ${ }^{1}$ Laboratorio de Paleontología, Facultad de Ciencias Marinas, Universidad Autónoma de Baja California, Ensenada, Baja California, \\ México, 22800. \\ ${ }^{2}$ Department of Vertebrate Paleotology, Natural History Museum of Los Angeles County, Los Angeles, California, U.S.A. 90007. \\ ${ }^{3}$ Departamento de Geología, Centro de Investigación Científica y Educación Superior de Ensenada C I C E S E, Carretera Ensenada- \\ Tijuana, Zona Playitas No. 3918, Baja California, México, 22860. \\ *aranda@uabc.edu.mx
}

\section{Resumen}

Los fósiles de cetáceos odontocetos del género Kentriodon son delfinoideos pequeños en la familia extinta Kentriodontidae. Este género ha sido reportado previamente en la Formación Rosarito Beach del Mioceno Medio en el área de La Misión en Baja California, México; sin embargo, los especímenes no habían sido descritos sistemáticamente. La especie de este depósito ha sido llamada aqui como Kentriodon diusinus, nueva especie, y su descripción está basada en elementos craneales y poscraneales. La especie pertenece a la subfamilia Kentriodontidae, y es la primera especie descrita para México. Un análisis filogenético realizado con el software PAUP* (Phylogeneic Analysis Using Parsimony and other methods) muestra que K. diusinus está relacionada más cercanamente con Kentriodon obscurus de la Fauna Local de Sharktooth Hill, derivada de Round Mountain Silt, Kern County, central California, EUA que con otras especies asignadas al género Kentriodon.

Palabras clave: Kentriodontidae, nueva especie, Mioceno Medio, Baja California, taxonomía.

\begin{abstract}
Fossil odontocete cetaceans of the genus Kentriodon are small delphinoid of the extinct family Kentriodontidae. This genus has been previously reported from the Middle Miocene Rosarito Beach Formation in the area of La Misión in Baja California, México; however, the systematics of the specimens have not been described previously. The species from this deposit is here named Kentriodon diusinus, new species, and is based on cranial and postcranial material. This species belongs in the subfamily Kentriodontinae, and it is the first described species of Kentriodontidae from México. A computer-assisted phylogenetic analysis using the software PAUP* (Phylogeneic Analysis Using Parsimony and other methods) shows that K. diusinus is most closely related to Kentriodon obscurus, which is known only from the Sharktooth Hill Local Fauna, derived from the Round Mountain Silt, in Kern County, central California, USA than any of the other species assigned to the genus Kentriodon.
\end{abstract}

Keywords: Kentriodontidae, new species, Middle Miocene, Baja California, taxonomy. 


\section{Introducción}

Los odontocetos fósiles del género Kentriodon (Kellogg, 1927), son delfines pequeños entre 2 y $5 \mathrm{~m}$ de largo, del Mioceno de la familia extinta Kentriodontidae. La familia está incluida en la Superfamilia Delphinoidea (Barnes, 1978, 1985). Dentro de la familia Kentriodontidae se reconocen cuatro subfamilias (Kampholophinae, Kentriodontinae, Pithanodelphininae, y Lophocetinae) y los miembros de estas subfamilias se conocen por fósiles descritos de Europa, Japón, Corea, Nueva Zelanda, y ambas costas de Norteamérica. (Aranda-Manteca, 1990, 2001; Barnes, 1977, 1978, 1985, 1988, 1997, 1998, 2002a, 2002b, 2002c; Barnes y Mitchell, 1984, 1985; Berta et al., 2006; Ichishima, 1995; Ichishima et al., 1995: figura 1b; Kázar y Venczel, 2003; Kazár, 2005; Kellogg, 1927, 1931, 1955; Lambert et al., 2005; Lim 2005; Rensberger, 1969).

Se ha hecho una revisión sistemática de la Subfamilia Kentriodontinae, en la que incluyen a tres especies descritas previamente dentro del género Kentriodon: Kentriodon pernix (Kellogg, 1927); Kentriodon obscurus (Kellogg, 1931); y Kentriodon hobetsu (Ichishima, 1995).

El género Kentriodon se ha reportado en depósitos Miocénicos de la Formación Rosarito Beach, en Mesa La Misión, cerca de La Misión en Baja California, México (Aranda-Manteca, 1990, 2001; Barnes, 1998; 2002c; Flores-Trujillo, 2002). Sin embargo, no se ha realizado una descripción a nivel de especie de los elementos de Baja California asignados a este género. En el presente trabajo se analizan los restos de un odontoceto colectado en la Mesa La Misión de la Formación Rosarito Beach que se encuentra almacenado en la Colección de Referencia del Estado de Baja California, Universidad Autónoma de Baja California, Facultad de Ciencias Marinas, Ensenada, Baja California, México. Aparte de la descripción de este espécimen se hace un primer análisis filogenético asistido por computadora usando el sotfware PAUP para poner en un contexto filogenético a este delfinoideo fósil.

\section{2. Área de estudio}

Los afloramientos rocosos de la Formación Rosarito Beach (Minch, 1967; Minch et al., 1970 y 1984) se encuentran distribuidos en la costa noroeste de la Península de Baja California, México, entre las ciudades de Tijuana y Ensenada (Figura 1). La Formación está dividida en dos subcuencas de depósito: la primera, al norte denominada Subcuenca Tijuana y la segunda al sur, conocida como Subcuenca la Misión. Esta última está constituida por una secuencia transgresiva-regresiva de capas intercaladas de sedimentos marinos y no marinos. Con base a sus litologías se han distinguido cinco miembros que conforman la Subcuenca la Misión: Miembro Punta Mesquite, Miembro

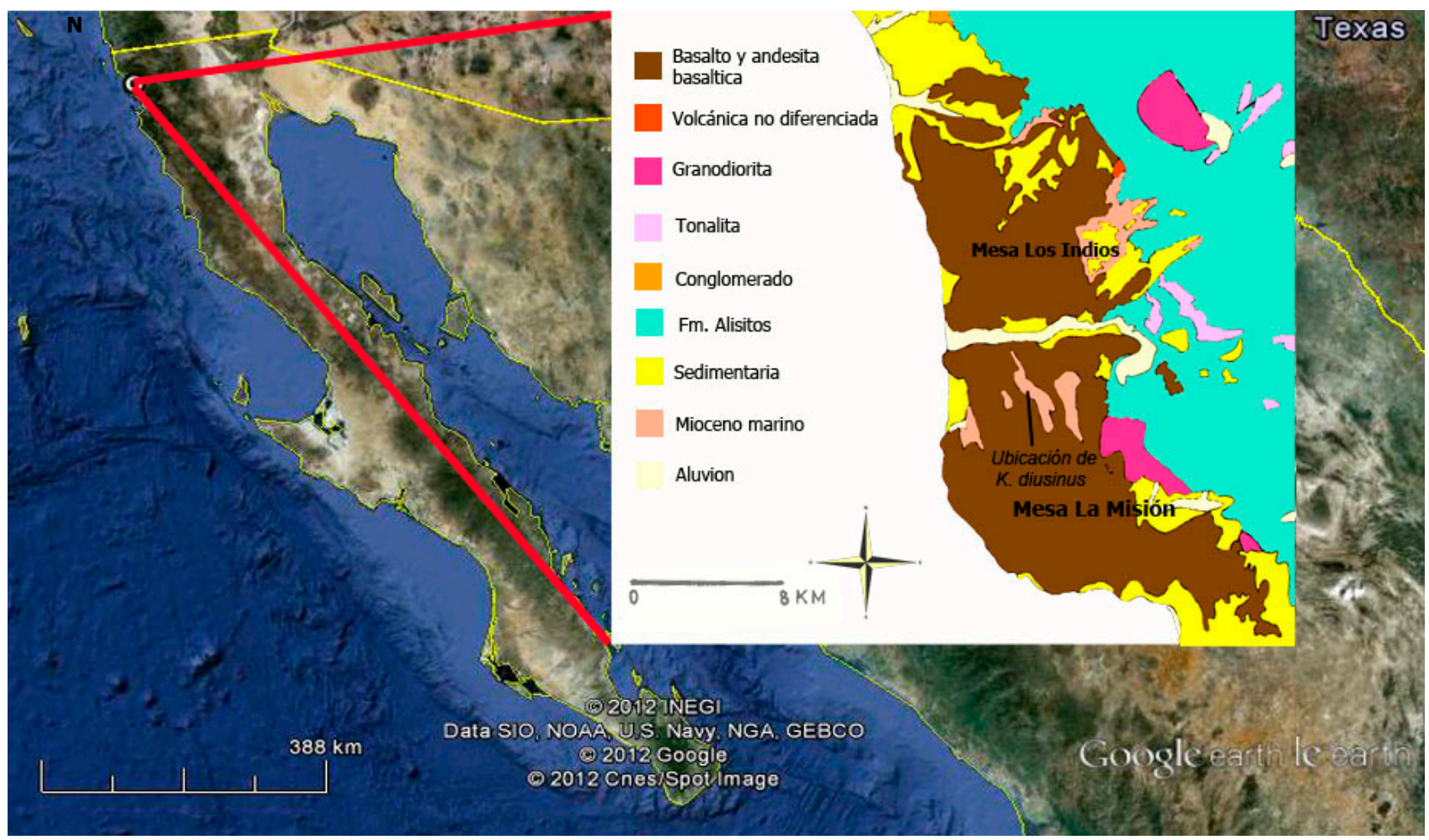

Figura 1. Mapa de la Zona de Estudio, (a) Vista aérea tomada de Google Earth con un polígono que delimita (b) la zona del mapa geológico. El punto amarillo en el mapa geológico indica el sitio de colecta de los restos de odontocetos analizados en este trabajo (Formación Rosarito Beach, Miembro los Indios, Mesa La Misión, localidad 103 capa G). Modificado de Sánchez-Gutiérrez, 2003. 
Medio Camino, Miembro La Misión, Miembro Los Indios y Miembro El Descanso (Ashby, 1989).

La zona de estudio es la Subcuenca La Misión, y está constituida por una secuencia transgresiva-regresiva de capas intercaladas de sedimentos marinos y no marinos. Con base a sus litologías se han distinguido cinco miembros que la conforman: Miembro Punta Mesquite, Miembro Medio Camino, Miembro La Misión, Miembro Los Indios y Miembro El Descanso (Ashby, 1989). El área de estudio corresponde al Miembro Los Indios. Este miembro aflora en las áreas al norte y sur del Arroyo Guadalupe. La localidad tipo fue descrita para la Mesa Los Indios en la región norte (Minch et al., 1984). La Mesa La Misión, al sur del Arroyo Guadalupe, muestra correlación con la estratigrafía de la localidad tipo, la cuál debe ser atribuida a la cercanía entre ambas localidades y concordancia de los procesos litogénicos (Aranda-Manteca, 2001). La localidad 103 de la Colección Paleontológica del Estado de Baja California se ubica al sur de la Mesa La Misión y está aflorando en el cañón más largo de la cuenca principal de drenaje de la Mesa. En esta localidad afloran las unidades denominadas C, D, E, F, G y H. La capa G es una arenisca tobácea, color gris claro a café anaranjado de $3.78 \mathrm{~m}$ de espesor, con sedimentos de arena fina. Esta capa contiene un horizonte de abundantes huesos (bonebed) que presentan evidencias de haber sido transportados (Aranda-Manteca, 2001). El organismo del presente trabajo fue colectado en esta capa.

El Miembro Los Indios en la Mesa La Misión, fue descrito por Minch y colaboradores (1970) y por sus características sedimentarias y fosilíferas, puede ser correlacionado con los estadíos bioestratigráficos de moluscos de la Fauna Temblor en Norteamérica, debido a la presencia de Turritela ocoyana, Anadara topagensis y Chione temblorensis (Addicott, 1972). También puede ser correlacionado con los estadíos bioestratigráficos de mamíferos continentales de Norteamérica, por la presencia del camélido Oxydactylus longipes (Minch et al., 1970). La presencia de diatomeas y silicoflagelados permiten correlacionar la secuencia de la Mesa La Misión con el estadío Luisiano de foraminíferos (Deméré et al., 1984), debido a la presencia del silicoflagelado de mar profundo Corbisema triacantha (Burky, 1975). Estas correlaciones corroboran los resultados obtenidos por fechado radiométrico de basaltos subyacentes a la secuencia sedimentaria (Gastil et al., 1975), que indican una edad de $16.1 \pm 2.1$ millones de años (Ma) para el basalto de La Misión y para el Miembro Costa Azul, que sobreyace al Miembro Los Indios, una edad de $14.3 \pm 2.6 \mathrm{Ma}$ (Hawkins, 1970). Basado en todo esto y con la asociación de vertebrados, en particular tiburones y mamíferos marinos (González-Barba, 1990; ArandaManteca, 1990), se atribuye el depósito al Mioceno Medio y puede correlacionarse con la localidad Sharktooth Hill (38 26'30'N, 118 $54^{\prime} 20^{\prime}$ 'W) de Round Mountain Silt en California, la Formación Calvert en Maryland y Virginia (Aranda-Manteca, 2001).

\section{Material y Métodos}

\subsection{Acrónimos}

FCMM: Colección de Referencia del Estado de Baja California, Ensenada, Baja California, México.

FCMM-A: Colección de Referencia del Estado de Baja California, Ensenada, Baja California, México. Mamíferos Marinos Actuales.

HMG: Hobetsu Museum, Hokkaido, Japón.

LACM: Department of Vertebrate Paleontology, Natural History Museum of Los Angeles County, Los Angeles, California, E.U.A.

U.S.N.M.: Division of Vertebrate Paleontology, United States National Museum, E.U.A.

UABC: Facultad de Ciencias Marinas, Universidad Autónoma de Baja California, Ensenada, Baja California, México.

\subsection{Medidas anatómicas y características}

Las medidas tomadas de los elementos de FCMM 2387 (Tabla 1) se hicieron basándose en la metodología descrita por Perrin (1975). Los huesos y estructuras anatómicas están señaladas de acuerdo a las siguientes abreviaturas: Aon, hendidura preorbital; Boc, basioccipital; Bs, basiesfenoides; C. oc, cóndilo occipital; Fo., foramen; Fr, frontal; Gl, fosa glenoide; La, lagrimal; Max, maxila; Na, nasales; Nar, narina; Pa, parietal; Pmx, premaxila; Poc, paraoccipital; Pr., proceso; Pro, preorbital; Psor, postorbital; Pt, Pterigoides; $\mathrm{Pt}(\mathrm{ML})$, Lámina media del pterigoides; Pts, fosa para el seno pterigoideo; Soc, supraoccipital; Sor, supraorbital; Vo, vómer; Zyg, zigomático.

\subsection{Análisis filogénetico}

Se realizó un análisis filogenético asistido por computadora usando el software PAUP versión $4.0 \mathrm{~b} 10$ (Swofford, 2002). La matriz contiene 15 caracteres craneales del espécimen Kentriodon diuisinus nueva especie, y 12 especies de odontocetos (nueve de estos han sido asignados a la familia Kentriodontidae, dos a Delphinidae y uno a la familia Squalodontidae) Los caracteres fueron codificados como binarios o multiestado. Para el carácter de "Vértice craneal" se creó una submatriz que impidiera el cambio de simetría derecha a izquierda y viceversa.

Los caracteres y estados de carácter se tomaron parcialmente de los trabajos de De Muizon (1988), Barnes (1990), Bianucci (2001, 2005), y Aguirre-Fernández et al., (2009). Algunos caracteres y estados de caracter fueron modificados y otros se proponen en el presente trabajo por primera vez (Tabla 2 y 3 ).

Para el análisis filogenético, se comparó el espécimen holotipo (FCMM 2387) de Kentriodon diusinus, nueva especie, con miembros de la familia Kentriodontidae: 
Kampholophus serrulus (Rensberger, 1969); Kentriodon hobetsu (Ichishima, 1995); Kentriodon obscurus (Kellogg, 1931); Kentriodon pernix (Kellogg, 1927); Liolithax kernensis (Kellogg, 1931); Liolithax pappus (Kellogg, 1955); Lophocetus repenningi (Barnes, 1978); Pithanodelphis nasalis (Barnes, 1985); Tagicetus joneti (Lambert et al., 2005), y usando al Squalodontoideo Zarhinocetus errabundus (Kellogg, 1931) como grupo externo (Fordyce y Barnes, 1994), con base en una revisión de las figuras del artículo en el que se describen por primera vez. También se analizaron directamente dos cráneos de miembros de la familia Delphinidae; del género Delphinus: Delphinus delphis (Linnaeus, 1758) (FCMM-A 1) y del género Tursiops: Tursiops truncatus (Montagu, 1821) (FCMM-A 2).

Se realizó un análisis de máxima parsimonia usando la opción de búsqueda de "branch and bound" del software PAUP, considerando todos los caracteres, exceptuando el 10, como ordenados (método Wagner). Esto significa que los estados de carácter están ordenados de acuerdo a su posición en la lista de símbolos, siendo el carácter codificado como 0 un estado ancestral, seguido de 1 como intermedio y 2 como derivado. El carácter 10, fue definido con una submatriz (stepmatix) que impide que la transformación del carácter de simetría craneal pase de ser simétrico izquierdo a simétrico derecho y viceversa; y que no se pueda regresar de un estado de asimetría a simetría. A los caracteres se les dio un peso de 100 pasos para caracteres binarios y de 50 pasos para los caracteres multiestado. La especie Zarhinocetus errabundus se definió a priori como grupo externo, asumiendo que todos sus caracteres son plesiomórficos (Barnes, 1985). La optimización de los estados de carácter fue deltran (transformación retrasada), dado que es más conservativa al asignar sinapomorfías a los grupos producto de una matriz de datos en la que hay un número sustancial de estados de caracter no determinados. El uso de este algoritmo para la optimización de caracteres favorece los paralelismos por sobre las reversiones (Wiley et al., 1991).

A los árboles resultantes del análisis por el método de Branch and Bound, se les aplicó un análisis de Bootstrap para asignar peso estadístico a la topología propuesta (Felsenstein, 1985; Shaffer et al., 1997), con los siguientes

Tabla 1. Medidas craneales de $K$. diusinus, nueva especie

\begin{tabular}{|c|c|}
\hline Medida & $\mathbf{c m}$ \\
\hline Longitud condilobasal & 33.4 \\
\hline Longitud de rostro & 22.5 \\
\hline Anchura de rostro en su base & 7.2 \\
\hline Anchura de rostro a $60 \mathrm{~mm}$ anterior a la línea que cruza los límites posteriores de las muescas anterorbitales & 3.5 \\
\hline Anchura del rostro a la mitad de su longitud & 2.3 \\
\hline Anchura de las premaxilas a la mitad de la longitud del rostro & 2.2 \\
\hline Anchura del rostro a 3/4 de la longitud, medido desde el extremo posterior & 17 \\
\hline Anchura preorbital máxima & 19.5 \\
\hline Anchura postorbital máxima & 14.8 \\
\hline Anchura supraorbital mínima & 12.5 \\
\hline Anchura máxima de las narinas externas & 2.4 \\
\hline Anchura máxima a través de los procesos zigomáticos del escamoso & 15.2 \\
\hline Anchura máxima de los premaxilares & 5.6 \\
\hline Anchura parietal máxima, dentro de las fosas postemporales & 14.9 \\
\hline $\begin{array}{l}\text { Altura vertical externa de la caja craneal desde la línea media del basiesfenoides al ápice del supraoccipital, pero } \\
\text { sin incluir la cresta supraoccipital }\end{array}$ & 3.5 \\
\hline Longitud máxima de la fosa postemoporal, medida al margen externo de la sutura elevada & 4 \\
\hline Anchura máxima de la fosa postemporal, medida al margen externo de la sutura elevada & 2.3 \\
\hline Diámetro máximo de la fosa temporal izquierda & 2.5 \\
\hline Diámetro mínimo de la fosa temporal izquierda & 3.1 \\
\hline $\begin{array}{l}\text { Distancia de los maxilares más allá de los maxilares, medidos desde la punta del rostro a la línea que pasa a } \\
\text { través de las puntas más cercanas de los maxilares que se puedan ver en vista dorsal }\end{array}$ & 2.1 \\
\hline Longitud del ápice en forma de órbita del proceso preorbital del frontal al ápice del proceso postorbital & 5 \\
\hline Longitud del proceso anterorbital del lacrimal izquierdo & 3.1 \\
\hline Anchura máxima de las narinas internas & 4.3 \\
\hline Longitud máxima del pterigoide izquierdo & 5.8 \\
\hline Longitud de la fila superior izquierda de dientes & 16 \\
\hline Número de dientes de la fila superior izquierda & 26 \\
\hline Número de dientes de la fila superior derecha & 26 \\
\hline
\end{tabular}

Según Perrin (1975) 
criterios:

100 réplicas con el criterio de parsimonia, búsqueda heurística y el algoritmo Tree-bisection-reconection (TBR), que dio lugar a un cladograma de consenso por regla mayoritaria con resolución al 50\% (Bootstrap 50\% majority-rule consensus tree) e incluyendo en el análisis a Zarhinocetus errabundus (Kellogg 1931), como taxón de referencia.

Las proporciones de bootstrap indican la frecuencia con que se encontraron los grupos en los árboles remuestreados con base en los resultados del análisis de branch and bound. Las proporciones de bootstrap (BPs), pueden interpretarse como: BPs $>90 \%$, evidencia de monofilia altamente significativa; BPs $89 \%-70 \%$ evidencia de monofilia marginalmente significativa; BPs $<70 \%$ evidencia de monofilia limitada (Shaffer et al., 1997).

\subsection{Especímenes de referencia}

El holotipo de Kentriodon diusinus, nueva especie, fue comparado morfológicamente con las figuras del holotipo de Kentriodon pernix (USNM 8060 y espécimen referido USNM 10670) (Kellogg, 1927), Kentriodon obscurus
(Kellogg, 1931) (LACM 21256) y Kentriodon hobetsu (Ichishima, 1995) (HMG 387) en donde aparecen publicados por primera vez.

\section{Resultados}

4.1. Sistemática

Clase Mammalia (Linnaeus, 1758)

Orden Cetacea (Brisson, 1762)

Suborden Odontoceti (Flower, 1867)

Superfamilia Delphinoidea (Gray, 1821; Flower, 1864)

Familia Kentriodontidae (Slijper, 1936; Barnes, 1978)

Subfamilia Kentriodontinae (Slijper, 1936)

Género Kentriodon (Kellogg, 1927)

Kentriodon diusinus, nueva especie.

\subsection{Sinónimos}

Kentriodon cf. K. obscurus. (Aranda-Manteca, 1990), ver Anexo 1.

Tabla 2. Definiciones de los caracteres morfológicos usados en el análisis filogenético.

\section{Rostrum}

1. Longitud del rostro: (0) muy alargado ( $>60 \%$ de CBL); (1) alargado (60-55\% CBL); (2) corto ( $<55 \% \mathrm{CBL})$. (D)

2. Premaxilas en el ápice del rostro: (0) se estrechan; (1) poseen márgenes laterales que se estrechan o divergen. (E)

3. Superficie dorsal de las premaxilas en la porción anterior del rostro: (0) abultada; (1) aplanada.(E)

4. Anchura premaxilar a la mitad del rostro en función de CBL: 80$)$ angosta $(<10 \%)$; (1) media $(10-15 \%$ CBL); (2) ancha (>15\%) CBL. (A).

5. Margen lateral del rostro: (0) cóncavo; (1) convexo (G).

6. Ancho del rostro a la mitad del rostro: (0) muy angosto ( $<10 \% \mathrm{CBL})$; (1) angosto (10-20\% CBL), (2) ancho $>20 \%$ CBL). (E).

\section{Neurocráneo}

7. Proceso anterorbital: (0) poco desarrollado; (1) cuadrangular; (2) triangular (G).

8. Hendidura anterorbital: (0) pequeña y amplia; (1) profunda y amplia; (2) profunda y angosta. (E).

9. Anchura zigomática como porcentaje de la longitud condilobasal: (1) menos de $45 \%$, (2) más de $45 \%$ (A).

10. Vértice craneal: (0) No torcido; (1) torcido a la derecha; (2) torcido a la izquierda. (B, D).

\section{Dientes}

11. Número de dientes por cada fila dental superior; (0) $>28$, (1) 28-20; (2)<20. (E).

12. Anchura del alveolo dental a la mitad del rostrum: (0) pequeño ( $<2 \% \mathrm{CBL})$; (1) grande $(\geq 2 \% \mathrm{CBL})$. (A).

\section{Seno ventral}

13. Crecimiento del seno aereo anterior: (0)no; (1) sí. (F, C).

14. Alargamiento del seno pterigoideo: (0) corto (razón largo/ancho <1); (1) mediano (razón largo/ancho 1-1.5); (2) largo (razón largo/ancho >1.5) $(\mathrm{G})$.

15. Ápice del seno pterigoideo: (0) redondeado; (1) punteado (F, D).

La codificación de los caracteres es: [0] postulado para caracteres considerados plesiomórficos; [1] caracter postulado como apomórfico; [2] carácter postulado como apomórfico alternativo. CBL significa longitud conbilobasal. (A) Aguirre-Fernández et al., 2009; (B) modificado de Barnes, 1990; (C) Bianucci, 1996; (D) Bianucci, 2005; (E) Modificado de Bianucci, 2005; (F) De Muizon, 1988; (G) Salinas-Márquez, 2011. 
Tabla 3. Matriz de caracteres que se utilizó para realizar el análisis filogenético.

\begin{tabular}{lcccccccccccccccc}
\hline \multicolumn{1}{c}{ Taxón } & $\mathbf{1}$ & $\mathbf{2}$ & $\mathbf{3}$ & $\mathbf{4}$ & $\mathbf{5}$ & $\mathbf{6}$ & $\mathbf{7}$ & $\mathbf{8}$ & $\mathbf{9}$ & $\mathbf{1 0}$ & $\mathbf{1 1}$ & $\mathbf{1 2}$ & $\mathbf{1 3}$ & $\mathbf{1 4}$ & $\mathbf{1 5}$ \\
\hline Zarhinocetus errabundus & 0 & 0 & 0 & 0 & 0 & 0 & 0 & 0 & 0 & 0 & 0 & 0 & 0 & 0 & 0 \\
Kentiodon hobetsu & 2 & $?$ & $?$ & 0 & $?$ & 0 & 1 & 1 & 0 & 0 & $?$ & $?$ & 1 & 0 & 0 \\
Delphinus delphis & 0 & 1 & 0 & 0 & 1 & 1 & 2 & 2 & 1 & 2 & 0 & 0 & 1 & 0 & 0 \\
Kampholophus serrulus & 0 & 0 & 0 & 0 & 0 & 1 & $?$ & 1 & $?$ & 0 & $?$ & $?$ & $?$ & $?$ & $?$ \\
Kentriodon diusinus & 1 & $?$ & 0 & 0 & 0 & 0 & 2 & 2 & 1 & 0 & 1 & 0 & 1 & 2 & 1 \\
Kentriodon obscurus & 0 & $?$ & $?$ & 0 & 0 & 0 & 1 & 2 & 1 & 0 & 0 & 0 & $?$ & 2 & 1 \\
Kentriodon pernix & 0 & 1 & 0 & 0 & 0 & 0 & 1 & 1 & 0 & 0 & 0 & 0 & 0 & 1 & 1 \\
Liolithax kernensis & 1 & 1 & 1 & 0 & 1 & 0 & 0 & 1 & $?$ & 2 & 0 & 0 & $?$ & $?$ & $?$ \\
Liolithax pappus & 0 & 0 & 0 & 0 & 0 & 0 & 1 & 2 & 0 & 0 & 1 & 0 & $?$ & 0 & 1 \\
Lophocets repenningi & 2 & 1 & 1 & 0 & 0 & 1 & 1 & 1 & 0 & 0 & 1 & 0 & $?$ & 1 & 1 \\
Pithanodelphis nasalis & 1 & 1 & 0 & 0 & 1 & 0 & 1 & 1 & 0 & 1 & 1 & 0 & $?$ & 1 & 0 \\
Tagictus joneti & 0 & 1 & 0 & 0 & 0 & 0 & 2 & 1 & 0 & 0 & 1 & 0 & 1 & 1 & 0 \\
Tursiops truncatus & 2 & 1 & 1 & 1 & 1 & 2 & 1 & 2 & 1 & 2 & 2 & 1 & 1 & 0 & 0 \\
\hline
\end{tabular}

La codificación de los caracteres son: [0] postulado para caracteres considerados plesiomórficos; [1] caracter postulado como apomórfico; [2] carácter postulado como apomórfico alternativo; y [?] cuando el carácter considerado no se ha preservado en el cráneo.

Kentriodon sp. (Aranda-Manteca, 2001), ver Tabla 10. aff. Kentriodon sp. (Barnes, 1998), ver Tabla 3. “... a skull of a small kentriodontine (in UABC FCM). (Barnes, 2002c):161.

"Kentriodontinael" (Flores-Trujillo, 2002), ver Anexo 1.

\subsection{Holotipo}

FCMM 2387, consiste de un cráneo, la parte posterior de las ramas mandibulares, un fragmento de la sínfisis mandibular, 2 dientes, el atlas, el axis, cinco vértebras verticales, dos vértebras torácicas (una completa y un centro vertebral) y la primer costilla derecha; todo pertenece al mismo organismo. Colector: Francisco Javier Aranda Manteca y Juan Gabriel Flores Trujillo, 1998.

\subsection{Horizonte y edad}

Formación Rosarito Beach, Miembro Los Indios, localidad FCMM 103 capa G. Mioceno Medio, 14-16 Ma (Aranda-Manteca, 2001).

\subsection{Diagnosis}

Kentriodon diusnus es una especie de Kentriodontidae que tiene un cráneo que difiere de K. pernix, K. obscurus, y $K$. hobetsu al tener un proceso preorbital más angosto, triangular y delgado dorsoventralmente; en tener los senos pterigoideos más alargados anteriormente sobre el paladar, que cualquier especie de Kentriodon descrito; en tener una tenue cresta en el supraoccipital que va desde la parte posterior de los frontales hasta el foramen magnum. Difiere aún más de K. pernix al tener un rostro más corto, frontales expandidos antero medialmente, las narinas son menos alargadas y más redondeadas; en tener un proceso paraoccipital proyectado más posteriormente. Difiere aún más de $K$. hobetsu en tener una hendidura preorbital mas angosta y marcada; en tener un cráneo con una forma más angular en lugar de redondeada; en tener premaxilas más angostas a la altura del supraorbital; en tener un borde lateral del supraorbital más cóncavo y tener un proceso postorbital visible dorsalmente; en tener parietales más grandes; difiere de $K$. obscurus al tener un rostro más angosto en la base y más delgado dorsoventralmente; en tener los palatinos y las fosas para los senos pterigoideos más alargadas anteriormente.

\subsection{Etimología}

El nombre diusinus, viene del latín diu que significa largo y de sinus que significa senos, haciendo alusión al tamaño de los senos pterigoideos de esta especie.

\subsection{Descripción}

\subsubsection{Cráneo}

Vista dorsal: El cráneo (Figuras 2a, 3a y 4a) está fracturado y comprimido dorsoventralmente y un poco hacia la derecha, debido a esto algunas partes se han deformado y otras están incompletas. El rostrum es un poco más largo que el largo del basicráneo y tiene una apariencia triangular. Las premaxilas están incompletas en la parte anterior siendo la derecha la más completa. La premaxila en la parte anterior del rostrum es elevada y parece divergir lateralmente en el ápice. A la altura de la hendidura preorbital, la premaxila está en posición horizontal y aplanada dorsoventalmente, se ensancha lateralmente y deja expuesto el surco mesorostral. Hay un foramen premaxilar en cada lado posterior a la hendidura preorbital. En ambos forámenes se observa un surco somero que va desde el foramen premaxilar hacia el borde lateral de cada premaxila con una ligera curva y otro que se extiende anteriormente en diagonal hasta llegar al surco mesorostral. Los bordes posteriores de las premaxilas se han perdido pero es posible distinguir la 
posición que tendría en la premaxila izquierda. El borde posterior de la premaxila parece ser delgado con una punta redondeada y llegar hasta el borde posterior de las narinas. Las maxilas también están incompletas, faltando el borde anterior, lo que impide conocer la longitud total real del cráneo. Ambas maxilas se encuentran juntas en el rostrum y se van engrosando lateralmente a medida que se acercan al basicráneo siendo visibles en vista dorsal en la mitad posterior del rostrum. En la maxila izquierda hay un foramen a la altura de la hendidura preorbital, este es grande y de forma ovalada. Posterior a la hendidura preorbital hay otro foramen de menor tamaño y de forma más bien circular. En la maxila derecha solo se distingue un pequeño foramen ovalado ligeramente posterior a la hendidura preorbital y muy cerca de la premaxila, debido a las fracturas es imposible identificar si hay un segundo foramen. No es posible identificar con claridad que tanto del proceso preorbital está cubierto por la maxila debido a la abrasión de esta estructura, pero es posible ver que la maxila llegaba hasta el borde lateral del supraorbital.

El borde posterior de la maxila se extiende hasta el escudo occipital, y medioposteriormente está en contacto con el frontal formando una pequeña cresta. Los nasales no
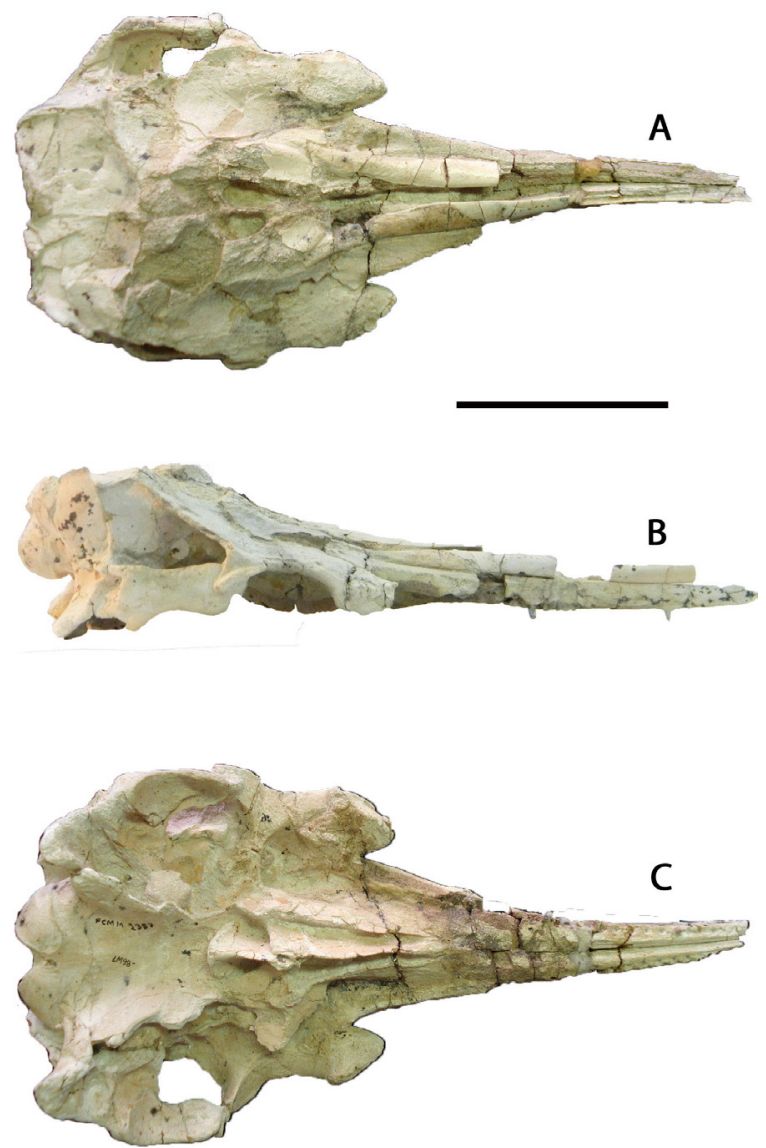

Figura 2. Kentriodon diusinus, nueva especie, holotipo, FCMM 2387, cráneo; A, vista dorsal; B, vista lateral; C, vista ventral; barra de escala a $10 \mathrm{~cm}$. están presentes y no se sabe cuánto contacto llegó a tener la maxila con estos huesos. El borde posterolateral de las maxilas es recto. La abertura nasal tiene una forma de gota, la parte anterior empieza en punta y el borde posterior está redondeado.

El proceso preorbital tiene una forma triangular con el borde anterior inclinándose ligeramente anteromedialmente. $\mathrm{La}$ hendidura preorbital tiene una forma en " $\mathrm{V}$ " bien marcada. El proceso posterorbital es agudo y, debido a la compactación del cráneo, se proyecta posterolateralmente. Es posible observar los procesos zigomáticos en vista dorsal, el proceso zigomático izquierdo se proyecta más lateralmente que el derecho debido a la compactación del cráneo; tienen una forma rectangular y llegan a tocar el proceso postorbital. El escudo occipital tiene una forma subrectangular con la parte anteromedial prolongada ligeramente anteriormente. Entre las maxilas y el escudo occipital los frontales aparecen con una forma romboidal con la esquina anteromedial y posterolateral prolongadas. En el escudo se alcanza a distinguir una cresta medial poco marcada. Los contactos con las maxilas, el frontal y los parietales están bien delimitados por crestas pronunciadas. Es posible ver parte de los cóndilos occipitales desde la vista dorsal.

Vista ventral: En el rostrum (Figuras 2b, 3b y 4b) la unión de las maxilas está marcada hasta los palatinos, en la parte más anterior forma un surco somero. Debido a la abrasión y a la fragmentación de las maxilas no es posible determinar el número exacto de alveolos dentales, pero el número parece estar entre 25 y 28. Los alveolos tienen un diámetro promedio de $3 \mathrm{~mm}$ y un espacio interalveolar de $4 \mathrm{~mm}$; quedan 2 dientes todavía en la maxila derecha. El borde de los palatinos no se distingue pero parece terminar muy cerca del borde anterior de los senos medios pterigoideos. La lámina media del pterigoides se ha inclinado hacia la derecha. El borde lateral derecho del pterigoides está inclinado a la derecha casi hasta una posición horizontal y cubre el canal orbital, del lado izquierdo, la lámina lateral del pterigoides se ha perdido. La parte posterior del vómer está levantada sobre el basioccipital debido a la compactación. En el proceso preorbital el lacrimal es grande y muy similar a Kentriodon pernix. El yugal se ha perdido por completo. El borde lateral del supraorbital tiene una forma cóncava y los procesos postorbitales se extienden más lateralmente que los preorbitales. Los procesos zigomáticos son cortos y la fosa glenoidea se inclina medialmente. El proceso falcado está inclinado posterolateralmente y se recurva lateralmente en el borde distal. Los procesos postoccipitales son pronunciados. El basioccipital es plano con dos ligeras depresiones anteriores a los cóndilos occipitales. Los huesos parietales son visibles a los lados de los cóndilos occipitales.

Vista lateral: Debido a los daños en el cráneo (Figuras 2c, $3 \mathrm{c}$ y $4 \mathrm{c}$ ), la descripción es una combinación de ambos lados. El rostrum es recto y se eleva suavemente a medida que se llega a la hendidura preorbital, siendo el punto más alto del cráneo los frontales. El escudo occipital es plano, esto es 

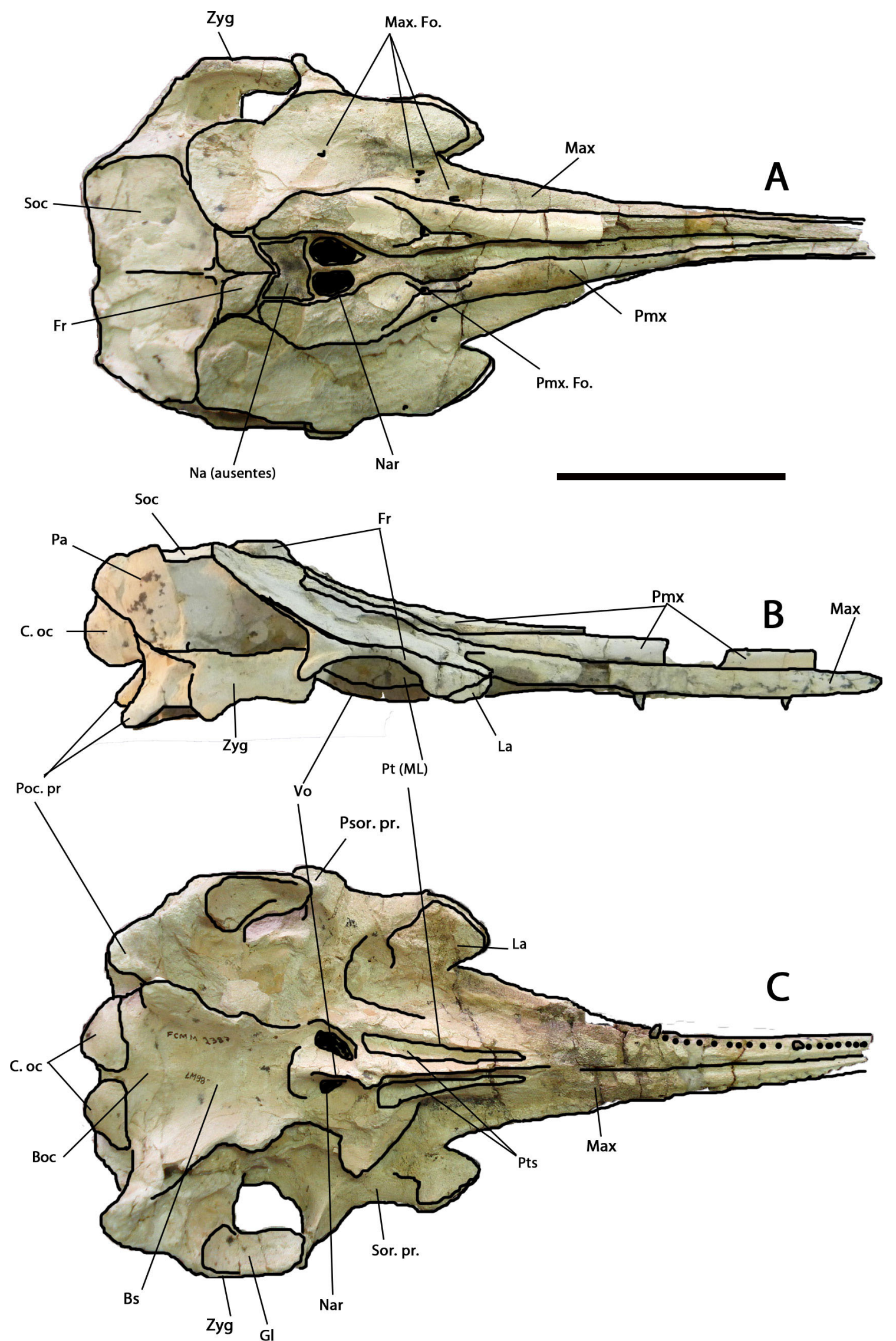

Figura 3. Kentriodon diusinus, nueva especie, holotipo, FCMM 2387, cráneo con partes indicadas y huesos delineados; A, vista dorsal; B, vista lateral; $\mathrm{C}$, vista ventral; ver metodología para la lista de abreviaciones; barra de escala a $10 \mathrm{~cm}$. 

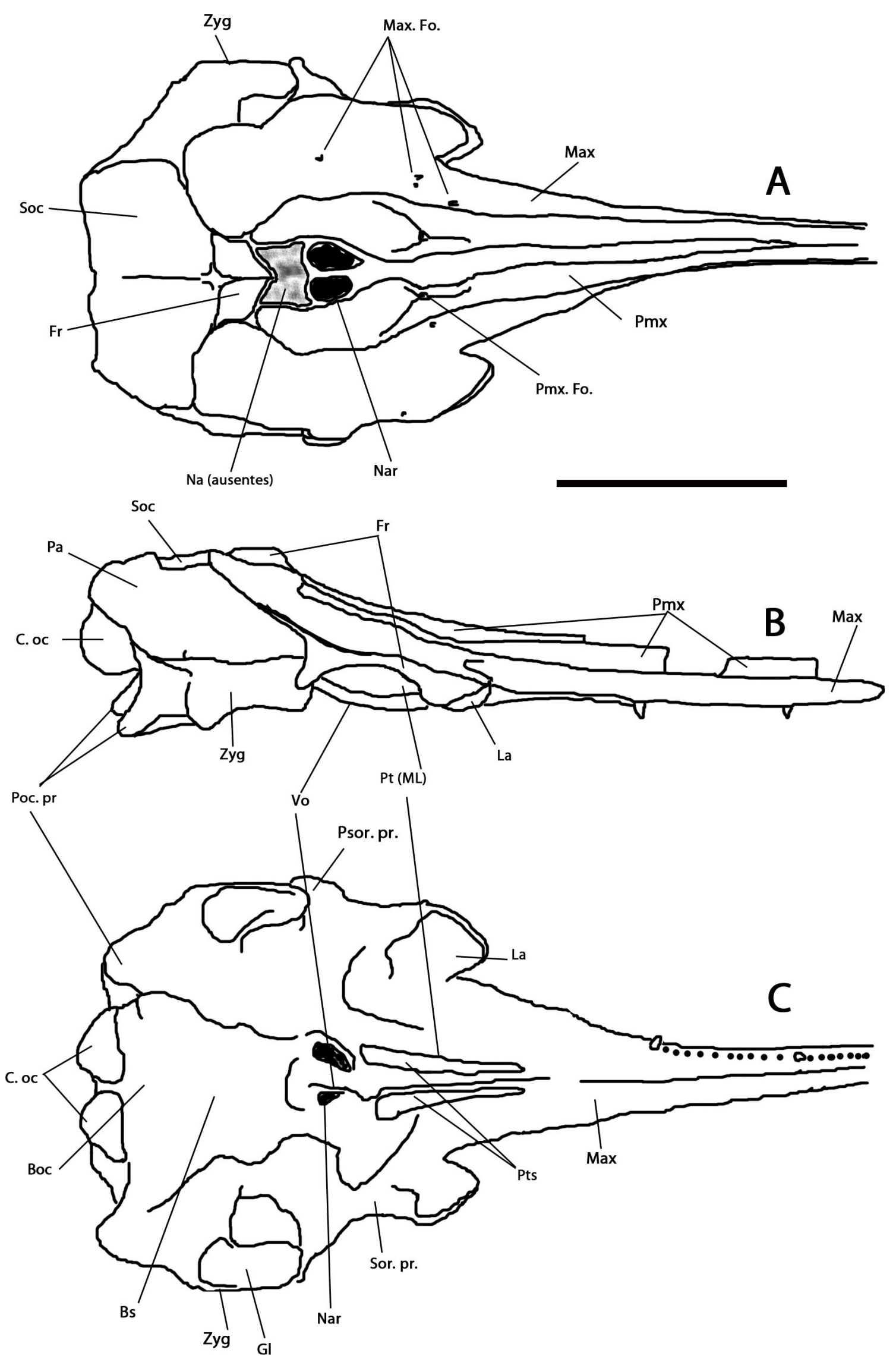

Figura 4. Kentriodon diusinus, nueva especie, cráneo del holotipo, FCMM 2387; A, vista dorsal; B, vista lateral; C, vista ventral. Delineados con partes indicadas y huesos delineados. Ver metodología para la lista de abreviaciones Barra de escala a $10 \mathrm{~cm}$. 
debido a la compactación, pero parece que la forma original no tenía una forma globosa sino ligeramente recurvada como en Kentriodon pernix. El proceso preorbital es grueso en su base y se vuelve más delgado en su parte anterior. El proceso postorbital se extiende posteoventralmente; este proceso tiene forma triangular y la punta termina en un ligero rectángulo en una posición más vertical que inclinada. El proceso zigomático está expandido dorsoventralmente y tiene una forma rectangular. Debido a la compactación, el proceso postorbital sobrelapa al proceso zigomático pero parece que no es así en vida. La cresta lambdoidal a pesar de estar fracturada presentan una inclinación caudomedial. La abertura formada por el proceso posterorbital y el zigomático tiene una forma casi triangular y extendida anteroposteriormente. El proceso postoccipital se proyecta ventroposteriormente y es el punto más bajo del cráneo.

\subsubsection{Mandíbula}

Ambos dentarios (Figura 5) están rotos y solo se conserva la parte posterior de cada rama mandibular y 4 $\mathrm{cm}$ de la parte anterior. El fragmento de la parte anterior de la mandíbula tiene 6 alveolos dentales en la parte derecha y 5 alveolos en la parte izquierda. De los 5 alveolos izquierdos se conservan en su sitio las raíces de 3 dientes. El fragmento, que comprende parte de la sínfisis, tiene una forma semicircular en vista anterior o posterior con un pequeño surco en la parte medial que se forma por la unión de ambas ramas mandibulares. En vista posterior es posible ver dos canales que corren por el centro de cada rama mandibular y llegan hasta donde estarían las raíces dentales. La parte posterior de ambas ramas mandibulares está muy fragmentada y solo se conservan algunos de los elementos. En la rama derecha es posible ver el proceso coronoide que se levanta con una suave pendiente, pero la parte posterior de este proceso no está conservado. En la rama izquierda el proceso coronoide se ha roto y no está unido al resto de la mandíbula. El cóndilo mandibular en ambas ramas está incompleto pero se conserva la parte lateral que es más gruesa y resistente que otras partes de la rama mandibular. Las fosas mandibulares se han roto y no es posible medir la distancia que hay entre ésta y el cóndilo mandibular.

\subsubsection{Dientes}

En la maxila, los dientes (Figura 6) tienen una forma cónica

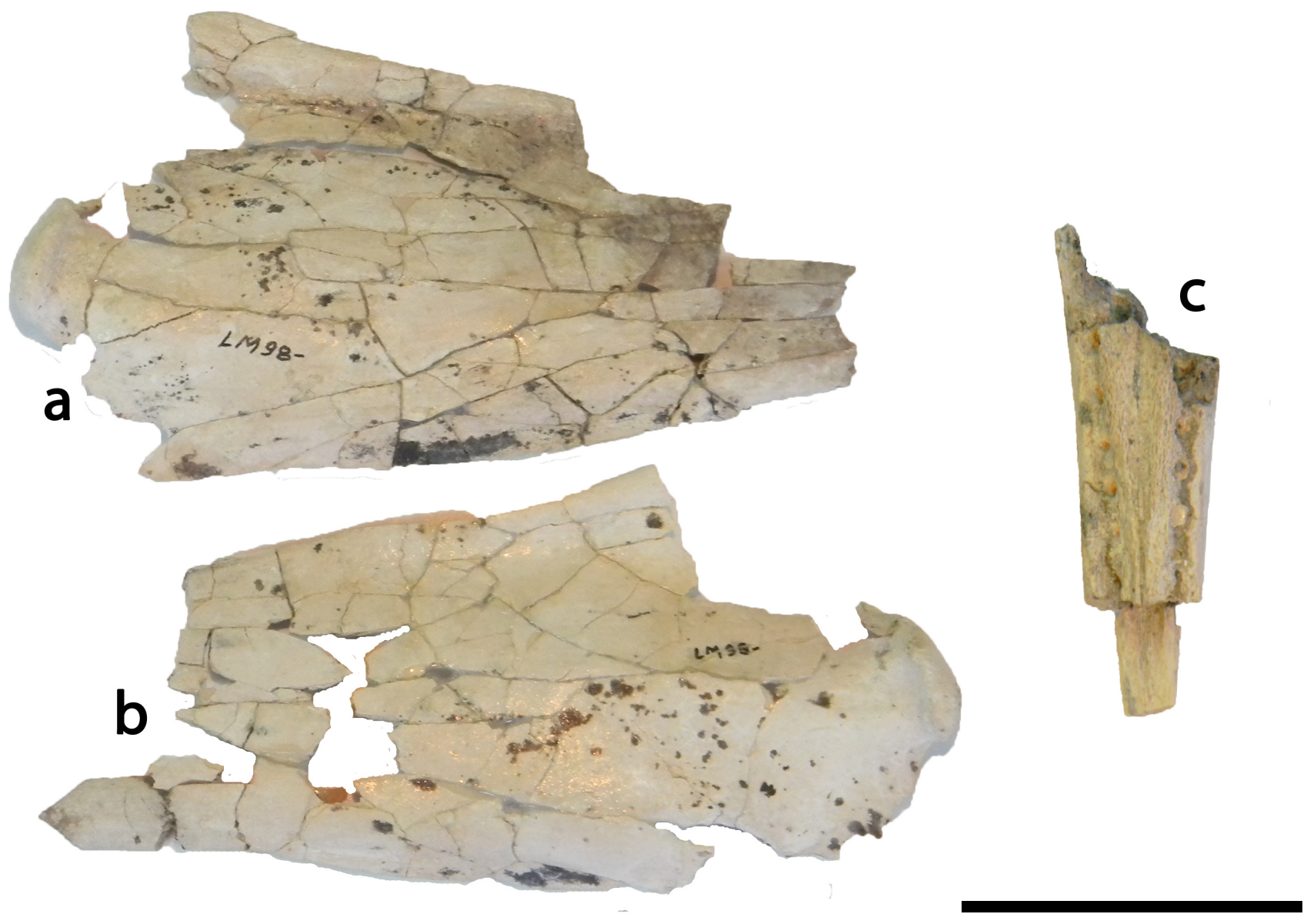

Figura 5. Vista lateral de la rama madibular derecha (a) e izquierda (b) y vista ventral del fragmento de la sinfisis mandibular (c). Barra de escala a $5 \mathrm{~cm}$. 
y están ligeramente comprimidos anteroposteriormente, así como están recurvados medialmente y miden $\sim 6 \mathrm{~mm}$ de la base de la corona a la punta. Aparte de estos dos dientes se tienen dos más que están sueltos, estos miden $15 \mathrm{~mm}$ de la raíz a la punta y la corona mide menos de un tercio de la longitud total del diente. La corona tiene una forma cónica con el ápice recurvado medialmente y no presenta cúspides accesorias.

\subsubsection{Atlas}

El atlas es pequeño siendo dos veces más alto que largo (Figura 7). La espina dorsal está muy poco desarrollada, los procesos inferiores son robustos y se proyectan lateroventralmente, mientras que los procesos superiores están rotos, así que no es posible saber que tanto se
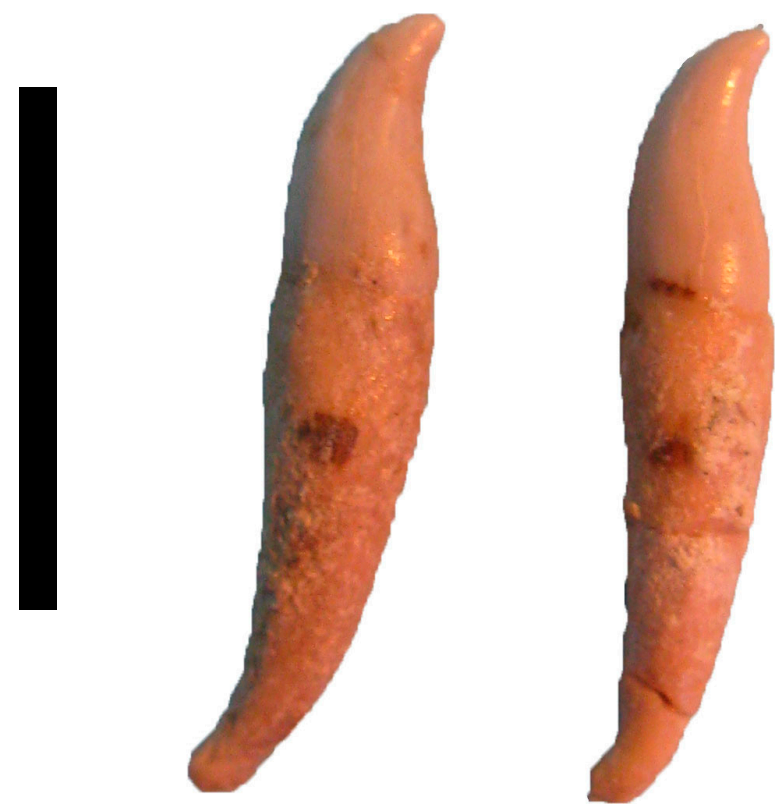

Figura 6. Dientes de Kentriodon diusinus, nueva especie. Barra de escala a $1 \mathrm{~cm}$. proyectaban. El arco neural es delgado anteroposteriormente. Las facetas anteriores son subovoidales siendo más anchas en la parte superior que en la inferior y están separadas por un arco amplio. El canal que se forma en el centro del atlas tiene una forma subovoidal y es amplio en la parte superior y un poco más angosta en la parte inferior. Las facetas posteriores son ligeramente cóncavas. El proceso hiapofisial es corto y grueso y en su parte superior hay una faceta ligeramente distinguible con una forma subtriangular para la articulación con el axis.

\subsubsection{Axis}

En el axis (Figura 8) la espina neural es gruesa e inclinada posteriormente y el arco neural forma un semicírculo. La faceta de articulación con el atlas del lado izquierdo se ha perdido y la derecha es ligeramente convexa. El proceso inferior derecho es grueso en la base y se va adelgazando a medida que llega a la parte más distal y está inclinado posteriormente. El centro del axis tiene una pequeña depresión en forma circular. Posteriormente, el centro del axis es cóncavo y la parte posterior del proceso inferior forma una cavidad subovoidal. En la parte inferior del axis hay dos depresiones que están separadas por un borde medial que corre anteroposteriormente a lo largo de toda la parte ventral.

\subsubsection{Tercera vértebra cervical}

Al igual que el cráneo, esta vértebra está compactada dorsoventralmente y ligeramente a la derecha (Figura 9a). Está fracturada y no se conserva el arco neural ni la espina neural. Tiene aproximadamente $1 \mathrm{~cm}$ de ancho, con procesos transversales inferiores aplanados y alargados anteroposteriormente. Los procesos transversales superiores se han roto pero parecen haber sido mucho más delgados. De los fragmentos restantes de los arcos neurales se observa que eran delgados y aplanados y le daban al canal neural una forma de riñón. En la tercera cervical del lado derecho se conserva la zigapófisis donde articula con el axis. Esta tiene una forma casi circular y ligeramente cóncava.

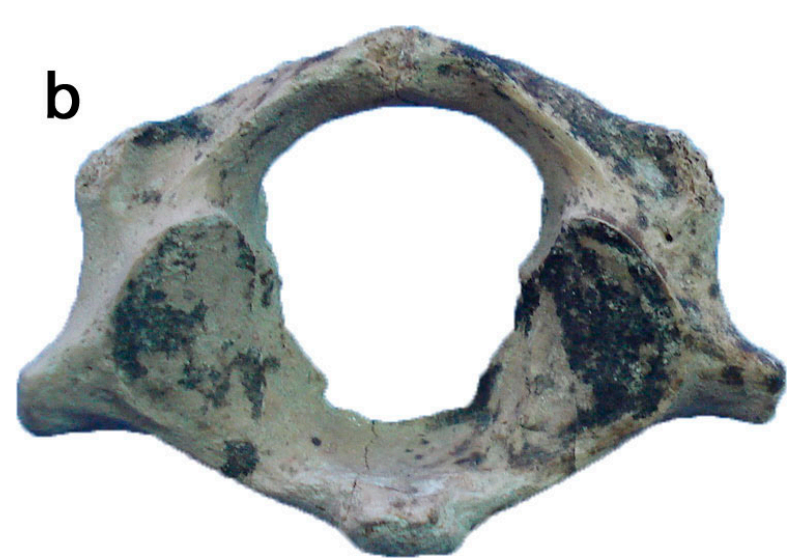

Figura 7. Vista anterior (a) y posterior (b) del atlas de Kentriodon diusinus, nueva especie. Barra de escala a $5 \mathrm{~cm}$. 

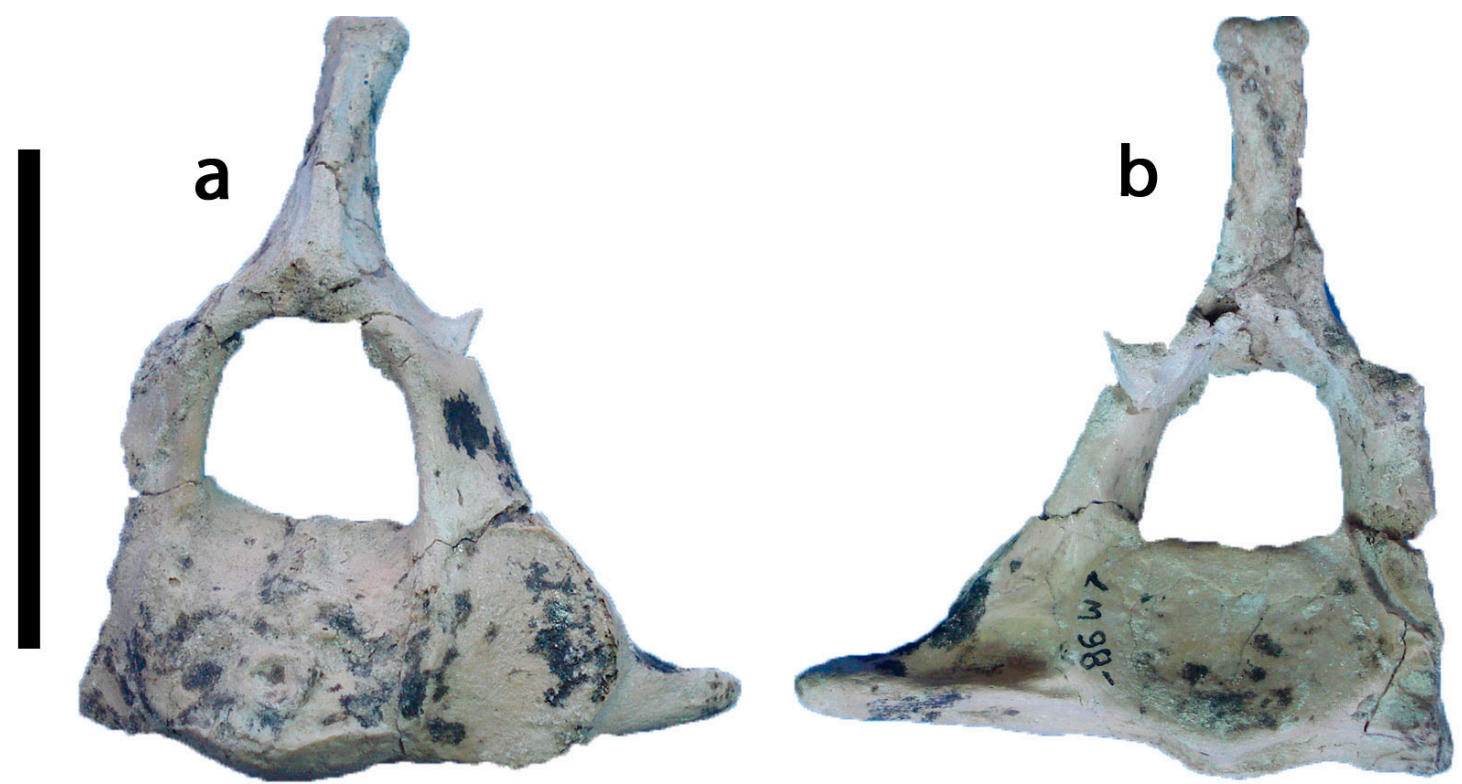

Figura 8. Vista anterior (a) y posterior (b) del axis de Kentriodon diusinus, nueva especie. Barra de escala a $5 \mathrm{~cm}$.
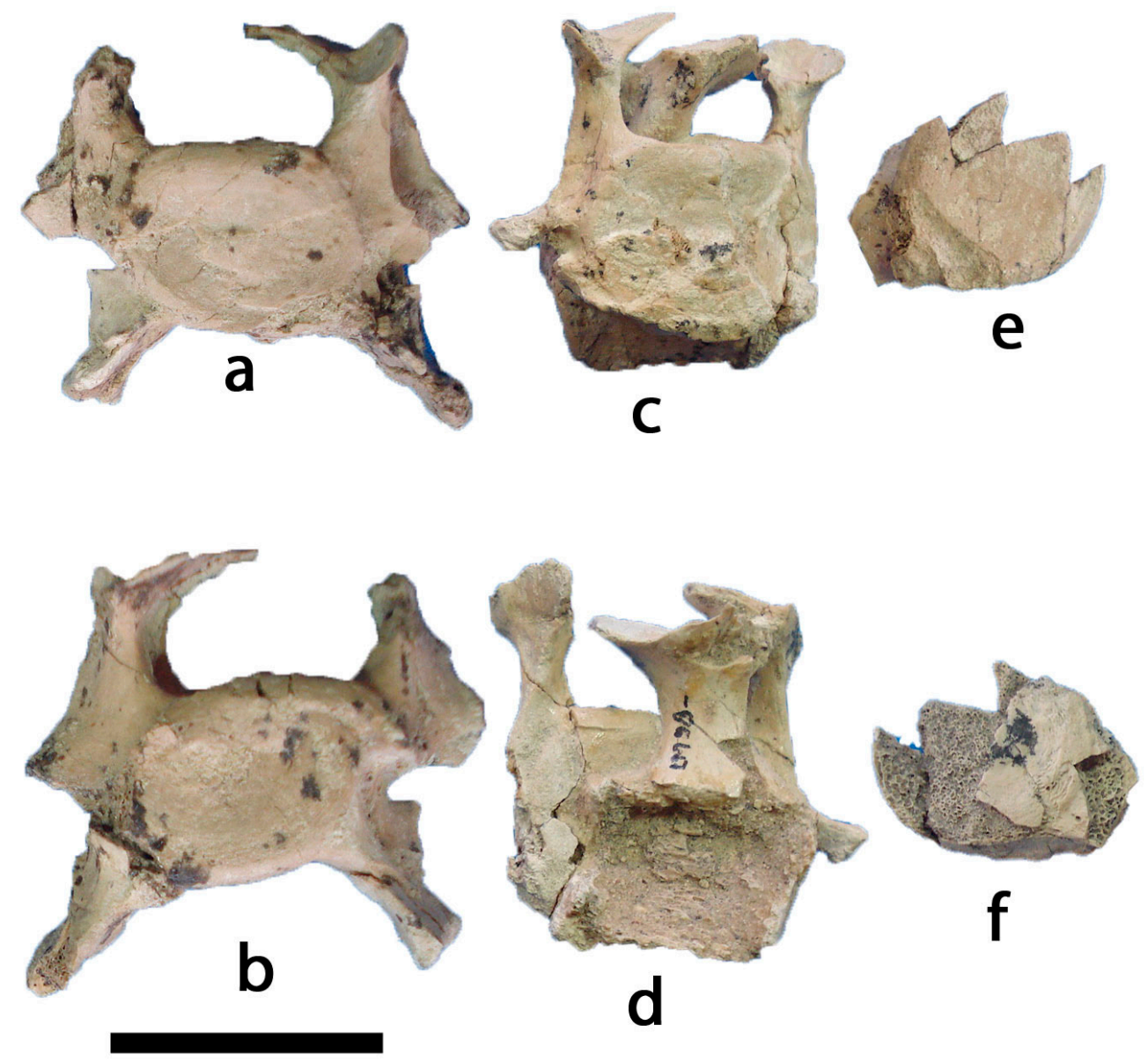

Figura 9. Vértebras cervicales. Vista anterior (a) y posterior (b) de tercera y cuarta cervical; vista anterior (c) y posterior (d) de quinta y sexta cervical; vista anterior (e) y posterior (f) de séptima cervical. Barra de escala a $3 \mathrm{~cm}$. 


\subsubsection{Cuarta vértebra cervical}

La cuarta vértebra cervical está fusionada con la tercera (Figura 9b). La forma es muy similar a la anterior en forma y estado de preservación. De las diferencias que se aprecian es que los procesos transversales son ligeramente más grandes y gruesos, y el borde dorsal del centro vertebral está más intemperisado que el de la tercera.

\subsubsection{Quinta vértebra cervical}

Al igual que las dos anteriores, los ejemplares también están unidos por sedimento y son imposibles de separar (Figuras 9c). La quinta vértebra cervical ha perdido la espina neural y la parte derecha del arco neural, así como los procesos transversales inferiores. La parte del arco neural que se conserva es delgado y aplanado con las zigapófisis un poco más grandes que en la tercera cervical, pero con la misma forma circular y ligeramente cóncava. Los procesos transversales superiores son muy delgados y cortos, inclinándose posteriormente y más anchos en el ápice que en la base. El centro de la vértebra tiene una forma ovalada que ha sido deformada por factores físicos.

\subsubsection{Sexta vértebra cervical}

La sexta vértebra cervical está mucho más dañada (Figura 9d). Sólo se conserva un pequeña parte del centro $\mathrm{y}$ un proceso transversal inferior. Este proceso es grueso, ligeramente aplanado dorsoventralmente y se ensancha en el ápice anteroposteriormente para formar una superficie plana de articulación con el proceso transversal inferior de la quinta y séptima vértebras cervicales.

\subsubsection{Séptima vértebral cervical}

De esta vértebra solo se conserva la mitad del cuerpo vertebral (Figuras 9e y 9f). Tiene aproximadamente el mismo grueso que las demás cervicales y parece que el proceso transversal inferior era igual de robusto al de las otras.

\subsubsection{Primera vértebra torácica}

El centro de esta vértebra es un poco más grueso que el centro de las cervicales (Figuras 10a y b). La espina neural es delgada lateralmente y se proyecta casi verticalmente. El arco neural es bajo, aplanado dorsoventalmente. Entre el proceso lateral y la espina neural hay una zigapófisis para la articulación con la segunda vértebra torácica. El proceso lateral es robusto y se proyecta desde la base del arco neural. Este proceso es subrectangular en el ápice y en la parte posterior tiene una faceta de forma triangular para la unión del tuberculum de la primera costilla.

\subsubsection{Segunda vértebra torácica}

De esta vértebra sólo se conserva el centro y la base de la
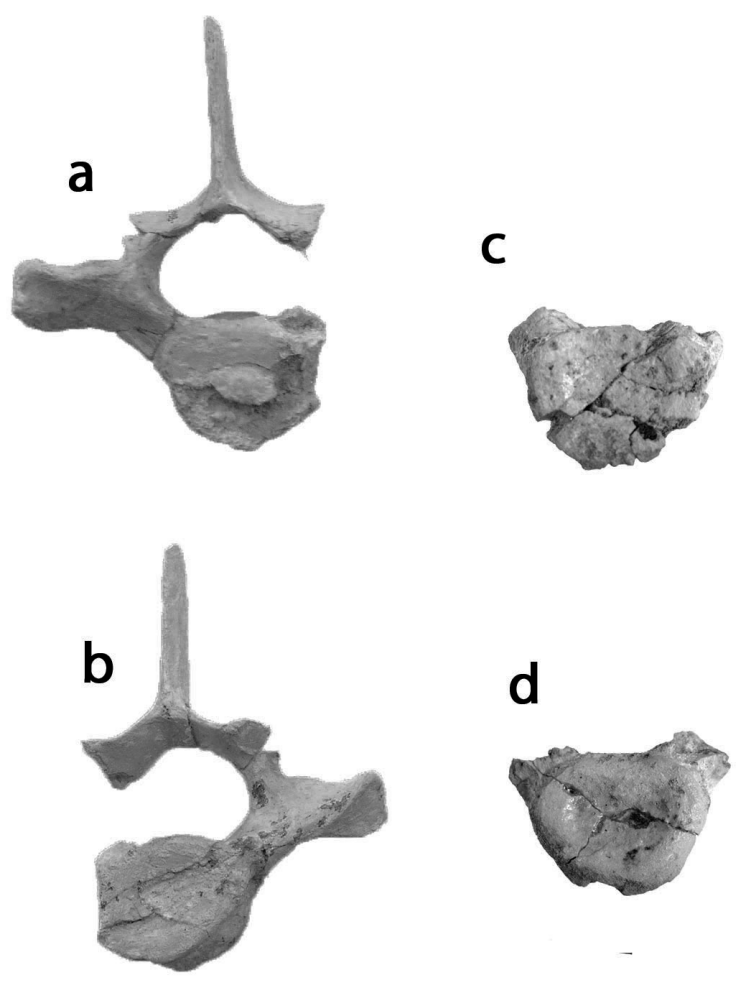
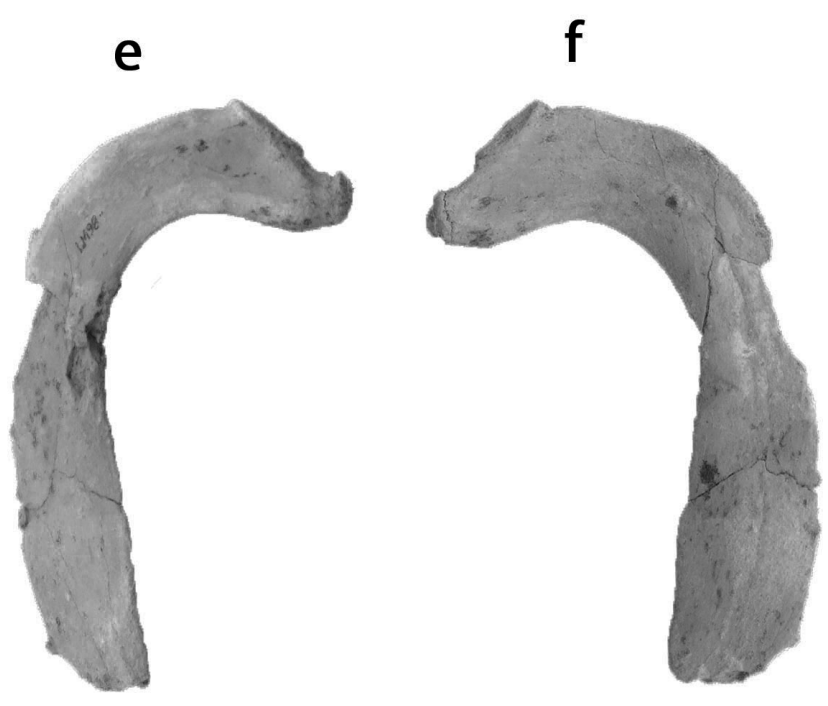

Figura 10. Primera vértebra torácica en vista anterior (a) y posterior (b); segunda vértebra torácica en vista anterior (c) y posterior (d); primera costilla derecha en vista (e) anterior y posterior (f). Barra de escala a $5 \mathrm{~cm}$. 
espina neural (Figuras 10c y 10d). El centro es parecido en tamaño y forma a la primera vértebra torácica, mientras que la espina es más ancha anteroposteriormente y parece que hubiera sido más larga que la espina de la primera vértebra torácica si se encontrara completa.

\subsubsection{Primera costilla derecha}

Esta costilla es corta y aplanada anteroposteriormente y no se mantiene sobre el mismo plano (Figuras 10e y 10f), la parte distal está torcida ligeramente hacia la parte anterior. En la parte proximal el capitulum es pequeño y bien marcado con una forma casi circular, después hay un espacio de $9 \mathrm{~mm}$ en el borde posterodorsal al que le sigue el tuberculum, una faceta que se une al proceso lateral de la primera vértebra torácica. El tuberculum tiene forma subtriangular y bien delimitada. En la parte anterior de la costilla, entre el capitulum y el tuberculum, hay una ligera depresión triangular de un par de centímetros de largo. La parte distal de la costilla tiene un borde recurvado y delgado anteroposteriormente y no parece haber una superficie de unión para la costilla esternal.

\subsection{Comparaciones}

Se asignó a FCMM 2387 al género Kentriodon ya que presenta características diagnósticas reportadas por Barnes (1978) y Barnes y Mitchell (1984): Tener un cráneo con rostrum largo, un margen lateral del supraorbital más cóncavo y superficies palatales de la maxila más transversalmente convexas que otros géneros de la familia, tener una fosa para el lóbulo postorbital del sistema de sacos aéreos del pterigoides en la superficie ventral del frontal, tener maxilas más separadas en el borde posterior $\mathrm{y}$ al mismo tiempo una mayor exposición de los frontales y tener un vértice craneal menos elevado que otros géneros. Otras características diagnósticas para el género no se pueden observar debido al estado de conservación del cráneo como: surco mesorrostral no cubierto por las premaxilas en el borde anterior, huesos nasales aplanados en lugar de bulbosos y tener los dos dientes más anteriores en la premaxila alargados en forma de pequeños colmillos apuntando anteriormente en la punta del rostro.

\subsubsection{Comparación de Kentriodon diusinus con Kentriodon pernix}

El cráneo del holotipo de Kentriodon diusinus nueva especie, (Figuras 11a y 11d, 12a y 12d), es casi del mismo tamaño que el cráneo de $K$. pernix, pero $K$. diusinus tiene un rostrum más largo y una mayor exposición de las maxilas en la parte dorsal del rostrum. De los cóndilos occipitales a la hendidura preorbital los cráneos tienen la misma longitud, pero el cráneo de $K$. diusinus es más ancho a la altura del arco zigomático. El proceso preorbital en $K$. diusinus es más prominente, tiene una forma subtriangular y proyectado anteriormente, mientras que en $K$. pernix es de menor tamaño, tiene una forma más cuadrangular y se proyecta más anterolateralmente. En ambos especímenes el borde lateral del supraorbital tiene una forma cóncava. El proceso zigomático es más grande en el cráneo de $K$. diusinus. Los frontales en el vértice craneal tienen una forma de gota en ambos especímenes, pero en K. pernix los bordes son más redondeados. Los bordes posteriores de la maxila se inclinan posteromedialmente en $K$. diusinus a diferencia de $K$. pernix en donde el borde posterior de la maxila se proyecta más posteriormente. En vista ventral, el pterigoides se proyecta más anteriormente en $K$. diusinus que en $K$. pernix, y la fosa glenoide del arco zigomático también es más pronunciada en $K$. diusinus que en $K$. pernix. Lateralmente, la fila de alveolos dentales de $K$. pernix termina más cercano a la hendidura preorbital que la fila dental de $K$. diusinus. El

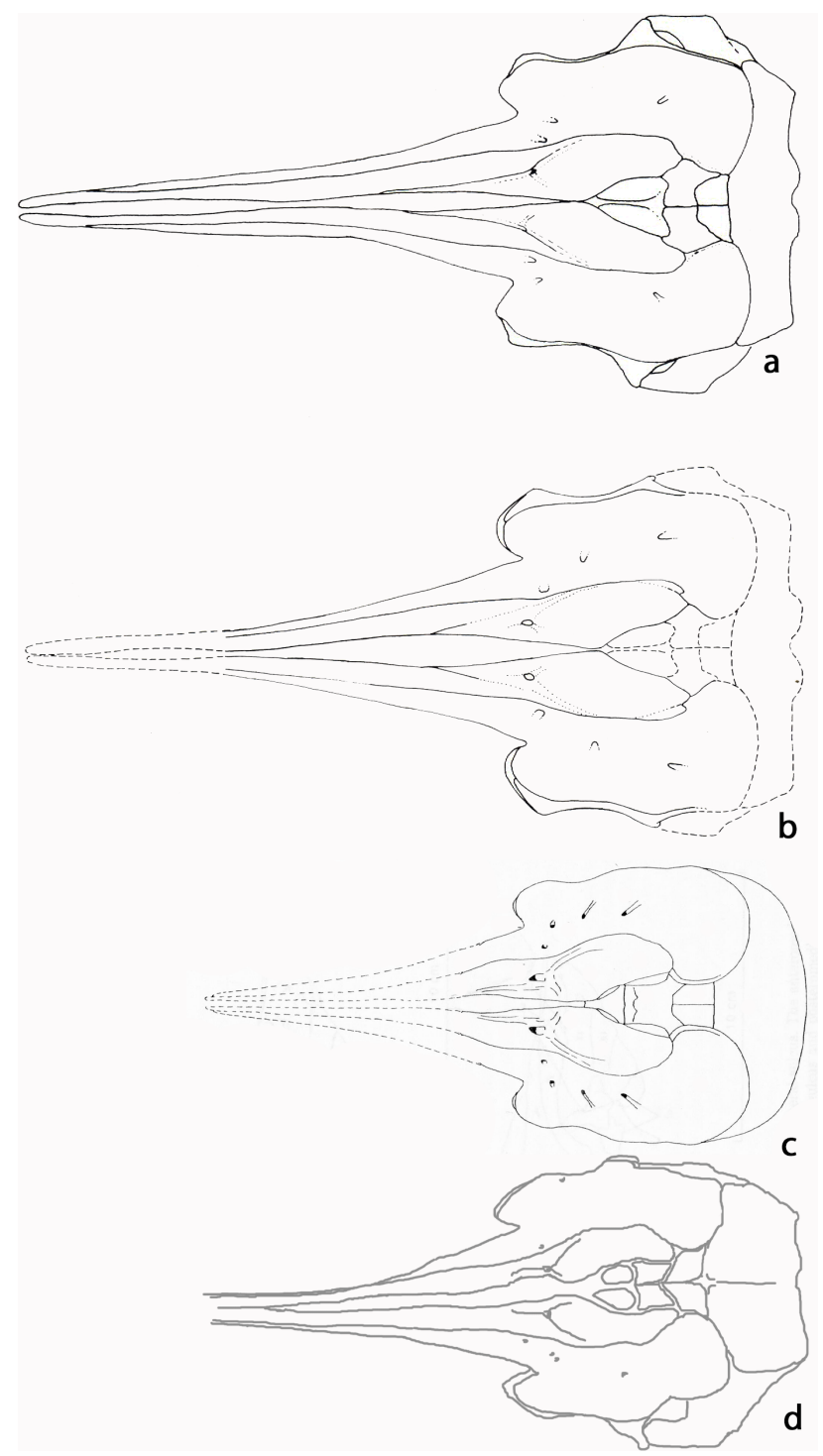

Figura 11. Delineados de Kentriodon pernix (a), Kentriodon obscurus (b), Kentriodon hobetsu (c) y Kentriodon diusinus, nueva especie. Las figuras no están a escala, se igualó la distancia del occipital a la muesca anterorbital. Figuras a y b tomadas de Barnes (1978), figura c modificada de Ichishima (1995). 
proceso preorbital es más grueso dorsoventralmente en Kentriodon pernix. El proceso postorbital en K. pernix está proyectado ventralmente, mientras que en $K$. diusinus se proyecta posteroventralmente. El borde lateral del frontal posterior al proceso postorbital es recto en $K$. diusinus y es ligeramente curvo en K. pernix. La superficie dorsal del supraoccipital es convexa en $K$. pernix y en $K$. diusinus es aplanado en la superficie dorsal del cráneo; sin embargo, esto puede ser un efecto de la compactación del cráneo y no ser una condición que hubiera presentado el organismo en vida. La cresta lambdoidal difiere entre los dos especímenes, en $K$. diusinus es recta y no se proyecta más allá de los cóndilos occipitales, mientras que en K. pernix la cresta lambdoidal es curva y se proyecta posteriormente más
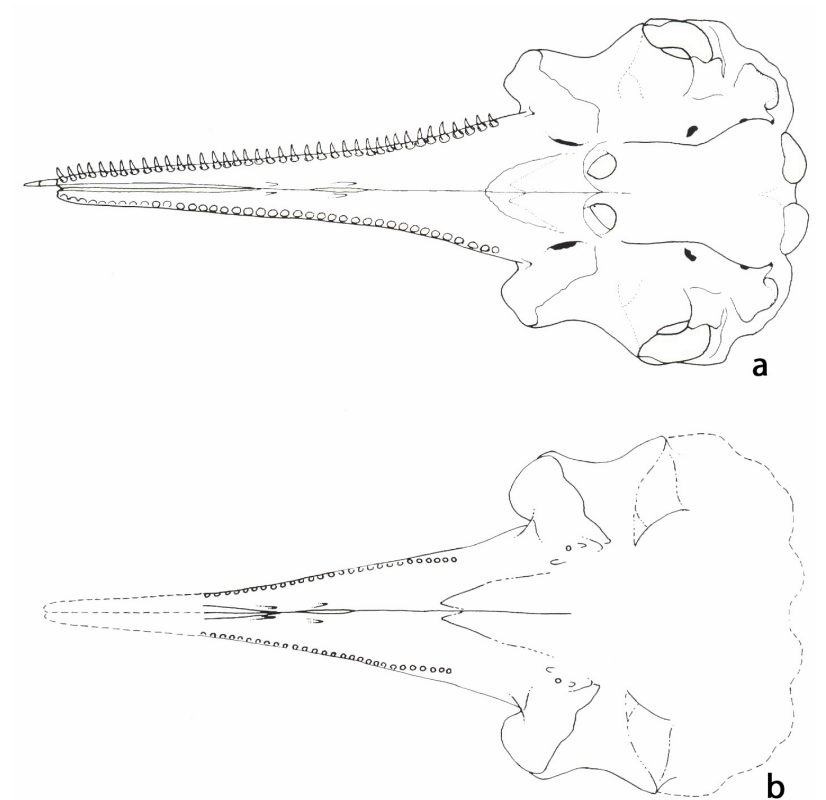

b
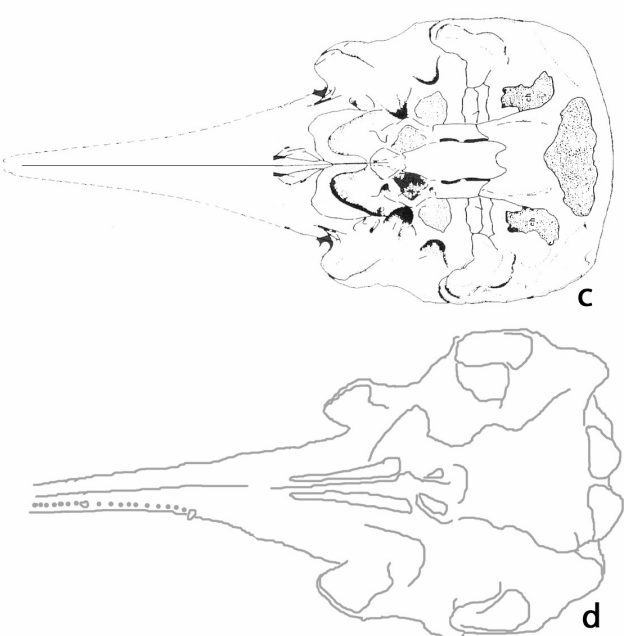

Figura 12. Delineados de la vista ventral de Kentriodon pernix (a), Kentriodon obscurus (b), Kentriodon hobetsu (c) y Kentriodon diusinus, nueva especie. Las figuras no están a escala, se igualó la distancia del occipital a la muesca anterorbital. Figuras a y b tomadas de Barnes (1978), figura c modificada de Ichishima (1995). allá de los cóndilos occipitales. El proceso paraoccipital en $K$. diusinus se proyecta posteroventralmente y en $K$. pernix la proyección es en dirección ventral. También la inclinación del proceso zigomático varía entre ambos, en $K$. diusinus está casi horizontal y en K. pernix se inclina anterodorsalmente.

\subsubsection{Comparación de Kentriodon diusinus con Kentriodon obscurus}

En primera instancia, el cráneo de $K$. diusinus presenta muchas similitudes con el de K. obscurus (Figuras $11 \mathrm{~b}$ y d, $12 \mathrm{~b}$ y $12 \mathrm{~d}$ ) y es al que más se parece de las tres especies de Kentriodon con las que se comparó el holotipo. Sin embargo, hay diferencias que impiden asignar el cráneo a esta especie. El rostrum es más ancho en $K$. obscurus a la altura de la hendidura preorbital. El foramen maxilar izquierdo se encuentra posterior a la hendidura preorbital en $K$. obscurus y anterior a ésta en $K$. diusinus. El proceso preorbital es triangular en $K$. diusinus y es trapezoidal en $K$. obscurus. Lateralmente, el rostrum es más grueso dorsoventralmente en $K$. obscurus, sin que exista evidencia de compactación, y el proceso postorbital se inclina ventralmente, mientras que en $K$. diusinus la inclinación es posteroventral. Los senos pterigoideos, que son la característica más relevante de este espécimen, también son largos con el borde anterior terminado en punta en $K$. obscurus pero no se proyectan tan anteriormente como lo hacen en $K$. diusinus.

\subsubsection{Comparación de Kentriodon diusinus con Kentriodon hobetsu}

En vista dorsal el ancho del rostrum a la altura de la hendidura preorbital es similar en ambos especímenes (Figuras 11c y 11d, 12c y 12d); sin embargo, la hendidura preorbital es más amplia en Kentriodon hobetsu y el proceso preorbital tiene una forma redondeada en $K$. hobetsu y es más bien triangular en $K$. diusinus. La forma del basicráneo en vista dorsal de $K$. hobetsu es redondeada, mientras que en $K$. diusinus tiene un basicráneo más angular. También, los procesos zigomáticos están visibles dorsalmente en $K$. diusinus, mientras que no son visibles en $K$ hobetsu. Los frontales en la porción del vértice craneal son subrectangulares en $K$. hobetsu y en $K$. diusinus tienen forma de gota, redondeados posterolateralmente y alargados anteromedialmente. Las premaxilas a la altura del supraorbital están más ensanchadas en $K$. hobetsu que en $K$. diusinus y el supraorbital es más cóncavo en $K$. diusinus. Lateralmente, se observa que el proceso preorbital en $K$. hobetsu es más grueso dorsoventralmente y el lagrimal está en una posición más inclinada. El hueso parietal difiere mucho en tamaño, siendo más pequeño en $K$. hobetsu. En vista ventral, la diferencia más resaltante es el tamaño y forma de los senos pterigoideos, son cortos con el borde anterior redondeado en $K$. hobetsu y en $K$. diusinus son muy alargados y se proyectan sobre el rostrum con el borde anterior terminado en punta. Otra diferencia es el tamaño de las fosas glenoides en el proceso zigomático, que son 
más cortas y con una constricción central en $K$. hobetsu lo que les da una apariencia de " 8 " y en $K$. diusinus son más alargadas y ovaladas.

\subsection{Análisis filogenético}

Del análisis filogenético se obtuvieron 26 árboles igualmente parsimoniosos de 2800 pasos cada uno (resultados no mostrados) pero las topologías variaban y los índices de consistencia y de retención presentaban valores bajos $(<0.60)$. Con el análisis de "Bootstrap" con 100 réplicas y usando la opción de algoritmo de cambio de ramas (Branch-swaping algorithm, TBR), se obtuvo un árbol de consenso con regla mayoritaria del $50 \%$. En el árbol obtenido, (Figura 13) solo se mantienen dos agrupaciones: una con Kentriodon obscurus y Kentriodon diusinus con una frecuencia de ocurrencia de $61 \%$, y el otro es el clado que agrupa a una politomía entre Liolithax sp., Delphinus delphis y Tursipos truncatus con una frecuencia de $64 \%$. Fuera de estos 2 clados, se mantiene con una frecuencia de $93 \%$, una politomía que incluye a todas las especies ocupadas en el análisis con excepción del grupo externo. Cuando se hace la reconstrucción de los caracteres se observa que los caracteres que agrupan a $K$. diusinus y $K$. obscurus son: una muesca aterorbital profunda y angosta, la anchura zigomática como porcentaje de la longitud condilobasal es mayor a $45 \%$, los senos pterigoideos son largos y el ápice del seno medio es punteado. Los caracteres que agrupan a Liolithax sp., Delphinus delphis y Tursiops truncatus son: margen lateral del rostro convexo, la anchura zigomática como porcentaje de la longitud condilobasal es mayor a $45 \%$, vértice craneal torcido hacia la izquierda y seno pterigoideo corto (Figura 14).

\section{Discusión}

\subsection{Filogenia}

No es la intención de este trabajo proveer con un análisis filogenético de la familia Kentriodontidae, ni una interpretación de las relaciones de los miembros de esta familia. Las hipótesis filogenéticas propuestas son simplemente para ubicar a Kentriodon diusinus en contexto con las otras especies fósiles para que en un futuro se pueda realizar un análisis más extensivo.

Los análisis filogenéticos de la familia son muy escasos y los trabajos sobre filogenia asistidos por ordenador son inexistentes. No se han definido caracteres específicos para la familia Kentriodontidae y la principal razón de esto es que no se tienen suficientes elementos craneales y no todos los que se tienen han sido descritos formalmente. Un ejemplo de esta problemática se presenta en Kellogg (1931) donde se describen 10 especies de delfinoideos con base en los

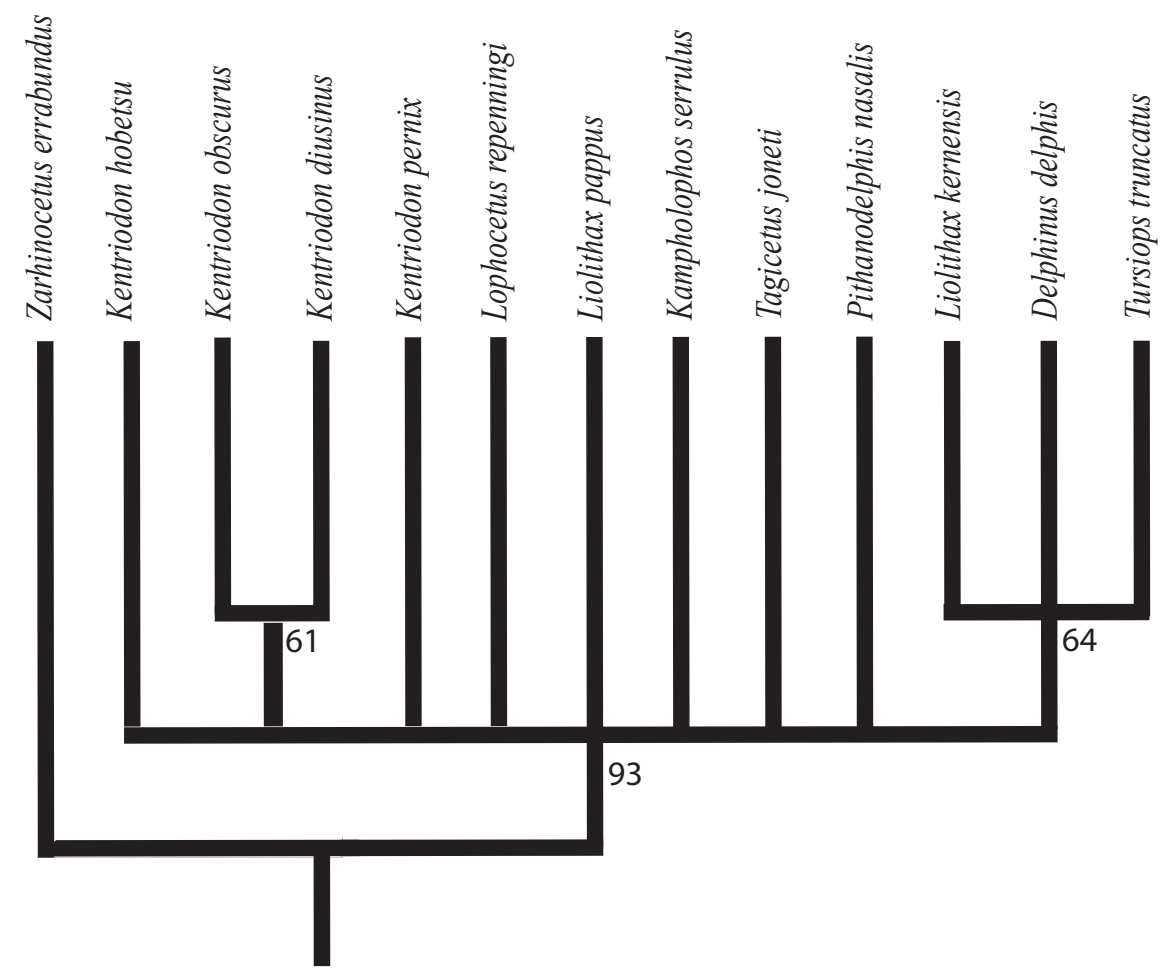

Figura 13. Cladograma creado por Bootstrap con regla mayoritaria al $50 \%$. Los números en la base de los nodos indican la frecuencia en porcentaje de aparición en las 100 repeticiones. Se incluyen en el análisis 10 miembros de la Familia Kentriodontidae incluyendo a Kentriodon diusinus, nueva especie, a 2 de la Familia Delphinidae y a uno de la Familia Squalodontidae como grupo externo. 
huesos perióticos únicamente. Al hacer esto se complica el análisis filogenético de estas especies debido a la falta de otras estructuras óseas.

Este trabajo es el primer acercamiento a un análisis basado en parsimonia para crear una clasificación. El haber obtenido índices de retención y consistencia bajos nos indica que hace falta aumentar los caracteres utilizados, o cambiar algunos de los caracteres propuestos para afinar la separación que existe entre las subfamilias y las especies dentro de estas subfamilias. Sin embargo, este análisis ha demostrado tener validez ya que en el análisis sí se hace una separación entre miembros de la familia Kentriodontidae y Delphinidae. También agrupa a $K$. diusinus y $K$. obscurus con base en la cantidad de similitudes craneales que presentan. Cabe mencionar que Liolithax kernensis no presenta características congenéricas con Liolithax pappus y se debe hacer una revisión más a fondo de estas dos especies (Flores-Trujillo et al., 2000; Barnes et al., 2008), lo que se refleja en el cladograma obtenido.

La agrupación de $K$. obscurus y $K$. diusinus que se había observado ya en el análisis de sus características morfológicas se mantiene en el cladograma de consenso estricto y en el cladograma generado por el Bootstrap, con un BPs de 61, lo que indica una monofilia limitada según Shaffer y colaboradores (1997).

Existe la posibilidad de que las diferencias entre $K$. diusinus y $K$. obscurus se deban a que sea la misma especie con dimorfismo sexual. Se ha observado variación en cachalotes, ballenas asesinas, ballenas con pico, narvales, belugas y ballenas piloto. Estas diferencias entre sexos ocurren en tamaño y forma de la cabeza, dientes, ancho torácico, aletas caudales, aletas pectorales, aleta dorsal, pedúnculo caudal joroba postanal y larga del rostro. En general, los machos tienden a tener apéndices de mayor tamaño que las hembras, la excepción son las especies en las que la hembra tiene un rostro más largo que el macho (Ralls y Mesnick, 2002). Sin embargo, no hay trabajos que se enfoquen en rasgos osteológicos en el dimorfismo sexual. Un análisis realizado en la morfometría craneal de diferentes cetáceos (Flores-Trujillo, 2002), que incluye cráneos de hembras y machos de la misma especie, muestra que las diferencias entre individuos de diferentes sexos son mínimas. Ya que las variaciones entre los cráneos de $K$. obscurus y $K$. diusinus no son solamente en la talla, sino también en el tamaño y la forma de diferentes estructuras craneales que no están relacionadas necesariamente con la reproducción, concluimos que es más probable que $K$. diusinus sea una especie diferente y no a dimorfismo sexual en la especie de $K$. obscurus.

\subsection{Morfología}

Las especies del género Kentriodon han sido reportadas para ambas costas de Estados Unidos, en Virginia, Maryland

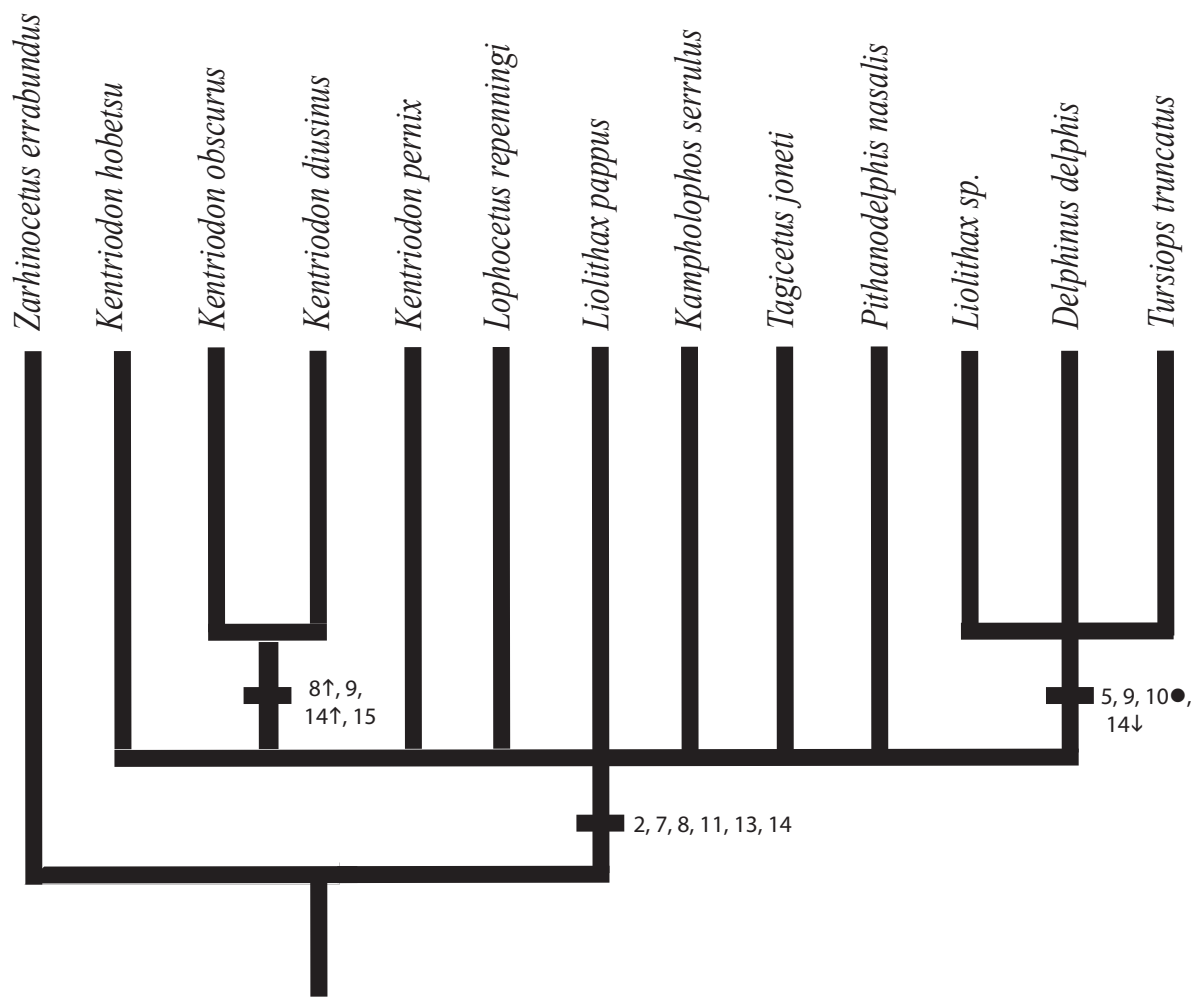

Figura 14. Cladograma que muestra la transformación de caracteres que generan las agrupaciones en el análisis de Bootstrap. Los números indican el paso de carácter de 0 a 1 , la $\uparrow$ indica una transformación de 1 a $2, \downarrow$ indica una regresión de 1 a 0 y $\bullet$ indica una transformación de 0 a 2 . 
y California, y Japón, en la región de Hokkaido (Ichishima et al., 1995); la presencia de K. diusinus marca el primer registro de este género en México y la distribución más sureña en el hemisferio norte.

Las características craneales que presenta el holotipo de Kentriodon diusinus, nueva especie, son congruentes para el género Kentriodon (ver Barnes, 1978). Así mismo, las características que presenta para la especie Kentriodon obscurus son muy similares a las características del holotipo de Kentriodon diusinus; sin embargo, si existen diferencias suficientes como para separarlo de esta especie: el supraorbital no es tan grueso ni arqueado como K. obscurus, el palatino y las fosas de los senos pterigoideos se extienden aún más anteriormente. La falta del hueso periótico en el holotipo de $K$. diusinus hace imposible una comparación de este elemento para esclarecer aún más las similitudes y diferencias entre estas dos especies.

Al comparar los dientes de $K$. diusinus con los dientes de Delphinus delphis se observó que la forma es muy similar, esto puede sugerir de un proceso que no necesariamente es evidencia de monofilia sino una simple convergencia heterocrónica. Se ha sugerido que algunos géneros de Delphinidae que tienen morfologías similares relacionadas con las estructuras de alimentación (proporciones del rostro, número y tamaño de los dientes) pudieron haberse originado independientemente por evolución convergente (Bianucci, 1996, 2005). Esto implicaría que $K$. diusinus pudo haber ocupado el mismo nicho ecológico que ocupa actualmente Delphinus delphis.

La madurez de un individuo puede estimarse con base en el grado de fusión de los elementos óseos craneales (Perrin, 1975; en Rommel y Reynolds, 2002). En el caso del holotipo de $K$. diusinus, los elementos craneales todavía no presentan una fusión total. Las premaxilas y las maxilas no están del todo fusionadas y las suturas todavía son visibles; los nasales no estaban fusionados con los frontales, esto inferido ya que los nasales no estaban presentes en el momento de la colecta del cráneo. Estas evidencias sugieren que el fósil perteneció a un organismo juvenil, pero tanto en las vértebras cervicales como en las vértebras torácicas, la fusión de los arcos neurales a los centros vertebrales es completa, lo que indica un adulto. Si tomamos en cuenta todos los elementos, llegamos a la conclusión de que se trata de un adulto joven.

\section{Conclusiones}

Una nueva especie de Kentriodon, Kentriodon diusinus, fue descrita a partir de un espécimen fósil. Este incluye un cráneo casi completo, las dos partes posteriores de la mandíbula, un pedazo de la sínfisis mandibular, dos dientes, las 7 vértebras cervicales, 2 vértebras torácicas, y una costilla.

Este espécimen fue colectado de la Formación Rosarito Beach, Miembro Los Indios, capa G, en Baja California,
México. El holotipo y único espécimen referido tiene una edad aproximada de 14-16 Ma. de antigüedad y corresponde con el periodo de Mioceno Medio.

Esta es la primera especie nombrada de Kentriodontidae para México, y es la especie más sureña que se ha encontrado para el hemisferio norte.

Kentriodon diusinus pertenece a la subfamilia Kentriodontinae y es muy semejante a Kentriodon obscurus, de Round Mountain Silt en California. Estas dos especies son muy cercanas tanto espacial como temporalmente pero las diferencias que presentan a nivel craneal son lo suficientemente marcadas como para considerarlas especies separadas.

El análisis filogenético ha mostrado validez al separar miembros de la Familia Delphinidae de Kentriodontidae, pero hace falta incluir más especies y hacer ajustes a la matriz de caracteres para que los resultados obtenidos sean estadísticamente más confiables.

\section{Agradecimientos}

Quiero agradecer a CONACyT por haberme otorgado una beca para la realización de mis estudios, a la National Geographic Society (Grant No. 5848-97, Middle Miocene Cetacea of La Mision, Baja California, México) que proporcionó los fondos para la extracción del material y a todas las personas que ayudaron a realizar la extracción y el preparado del material y que este trabajo fuera posible.

\section{Referencias}

Addicott, W.O., 1972, Provincial middle and late Tertiary molluscan stages, Temblor Range, California, Proceedings of the Pacific Coast Miocene Biostratigraphic Symposium, en Stinemeyer, E.H. (ed.), Bakersfield, California: Society of Economic Paleontologists and Mineralogists, 1-26.

Aguirre-Fernández, G., Barnes, L.G., Aranda-Manteca, F.J., FernándezRivera, J.R., 2009, Protoglobicephala mexicana, a new genus and species of Pliocene fossil dolphin (Cetacea; Odontoceti; Delphinidae) from the Gulf of California, Mexico: Boletín de la Sociedad Geológica Mexicana, 61(2), 245-265.

Aranda-Manteca, F.J., 1990, Aspectos paleoceanográficos y paleoecológicos de los fósiles del Mioceno, la Mesa La Misión, Baja California, México: Revista de la Sociedad Mexicana de Paleontología, 3(1), 97-116.

Aranda-Manteca, F.J., 2001, Análisis paleoecológico y paleoambiental de la asociación de vertebrados marinos fósiles de La Misión, Baja California, México: Universidad Autónoma de Baja California, Facultad de Ciencias Marinas, tesis de Doctorado, 211 p.

Ashby, J.R., Jr., 1989, Miocene tectonostratigraphic history of the Rosarito Beach Basin, northwestern Baja California: Implications for the early tectonic development of the southern California Continental Borderland: University of California at Los Angeles, tesis de maestria, $149 \mathrm{p}$.

Barnes, L.G., 1977, Outline of eastern North Pacific fossil cetacean assemblages: Systematic Zoology, 25(4), 321343.

Barnes, L.G., 1978, A review of Lophocetus and Liolithax and their relationships to the delphinoid family Kentriodontidae (Cetacea: Odontoceti): Natural History Museum of Los Angeles County Science Bulletin, 28, 1-35. 
Barnes, L.G., 1985, The Late Miocene dolphin Pithanodelphis Abel, 1905 (Cetacea: Kentriodontidae) from California: Contributions in Science, Natural History Museum of Los Angeles County, 367, 1-27.

Barnes, L.G., 1988, A Late Miocene dolphin, Pithanodelphis nasalis, from Orange County, California, en Koerper, H.C. (ed.), The Natural and Social Sciences of Orange County, Memoirs of Natural History Foundation of Orange County, vol. 2: Huntington Beach, California, 7-21.

Barnes, L.G., 1990, The fossil record and evolutionary relationships of the genus Tursiops, en Leatherwood, S., Reeves, R.R. (eds.), The bottlenose dolphin: San Diego, California, Academic Press, Inc., Chapter 1, 3-26.

Barnes, L.G., 1997, The fossil marine mammals of Mexico, $7^{\text {th }}$ International Theriological Congress, Abstracts of Oral and Poster Papers: Acapulco, México, September 6-11, 1997, 39-40

Barnes, L.G., 1998, The sequence of fossil marine mammal assemblages in México, en Carranza-Castañeda, O., Córdoba-Méndez, D.A. (eds.), Avances en Investigación Paleontología de Vertebrados. Publicación Especial 1, Instituto de Investigaciones en Ciencias de la Tierra: Universidad Autónoma del Estado de Hidalgo, Pachuca, Hidalgo, México, 26-79.

Barnes, L.G., 2002a, Cetacea, Overview, en Perrin, W.F., Thewissen, H., Wursig, B. (eds.), Encyclopedia of Marine Mammals: Academic Press, 204-208.

Barnes, L.G., 2002b, Delphinoids: Evolution of the modern families, en W.F. Perrin, H. Thewissen, Wursig, B. (eds.), Encyclopedia of Marine Mammals: Academic Press, 314-316.

Barnes, L.G., 2002c, Evolutionary history of the fossil marine mammals of México, en Montellano-Ballesteros M., Arroyo-Cabrales, J. (eds.), Avances en los estudios paleomastozoologicos, Colección Científica, Serie Arqueología: Instituto Nacional de Antropologia e Historia, México, D.F., México, 125-225.

Barnes, L.G., Aranda-Manteca, F.J., 1997, New Middle Miocene Paleoparadoxidae (Mammalia, Desmostylia) from Baja California and California: Journal of Vertebrate Paleontology, 17(supplement to 3 ), 30A-31A.

Barnes, L.G., Domning, D.P., Ray, C.E., 1985, Status of studies on fossil marine mammals: Marine Mammal Science, 1(1), 15-53.

Barnes, L.G., Mitchell, E.D., 1984, Kentriodon obscurus (Kellogg, 1931), a fossil dolphin (Mammalia: Kentriodontidae) from the Miocene Sharktooth Hill bonebed in California: Contributions in Science, Natural History Museum of Los Angeles County, 353, 1-23.

Barnes, L.G., Thomas, H.W., McLeod, S.A., Bohaska, D.J., Godfrey, S.J., Pyenson, N.D., Goedert, J.L., Aranda-Manteca, F.J., AguirreFernandez, G., 2008, Evolutionary diversity and determination of character polarity in primitive Miocene delphinoids, the Kentriodontidae (Cetacea, Odontoceti, Kentriodontidae): Programa y Resúmenes, XXXI Reunión Internacional sobre el Estudio de los Mamíferos Marinos, Ensenada, Baja California, Mexico, 18-21.

Berta A., Sumrich, J.L., Kovacs, K.M., 2006, Cetacean Evolution and Systematics, en Berta, A., Sumrich, J.L., Kovacs, K.M., (eds.), "Marine Mammals: Evolutionary Biology": Elsevier Inc. San Diego, CA., 51-82.

Bianucci, G., 1996, The Odontoceti (Mammalia, Cetacea) from Italian Pliocene. Systematics and phylogenesis of Delphinidae: Palaeontographia Italica, 83, 73-167.

Bianucci, G., 2001, A new genus of kentriodontid (Cetacea: Odontoceti) from the Miocene of South Italy: Journal of Vertebrate Paleontology, 21(3), 573-577.

Bianucci, G., 2005, Arimidelphis sorbinii a new small killer whale-like dolphin from the Pliocene of Marecchia River (Central Eastern Italy) and a phylogenetic analysis of the Orcininae (Cetacea: Odontoceti): Rivista Italiana di Paleontologia e Stratigrafia, 111(2), 329-344.

Burky, D., 1975, Cocolith and silicoflagellate Stratigraphy, Northwestern Pacific Ocean, Deep Sea Drilling Project, Leg 29: Innitial reports of the Deep Sea Drilling Project. 29 Innitial reports of the Deep Sea Drilling Project, U.S.A. 29, 845-872.

De Muizon, C., 1988, Les relations phylogénétiques del Delphinida
(Cetacea, Mammalia): Annales de Paléontologie, 74(4), 159-227.

Deméré, T.A., Roeder, M.A., Chandler, R.M., Minch, J.A., 1984 Paleontology of the Middle Miocene Los Indios Member of the Rosarito Beach Formation, northwestern Baja California, México, en Minch, J.A., Ashby, J.R. (eds.), Miocene and Cretaceous Depositional Environments, Northwestern Baja California, México: American Association of Petroleum Geologists, Pacific Section, $54,47-56$.

Felsenstein, J., 1985, Confidence limits on phylogenetics: An approach using bootstrap: Evolution, 39, 783-791.

Flores-Trujillo, J.G., 2002, Morfología y Morfometría del Cráneo y del Oído Interno (Hueso Periótico) en Odontocetos Delphinoidea: Universidad Autónoma de Baja California, tesis de Maestría, 168 p.

Flores-Trujillo, J.G., Aranda-Manteca, F.J., Barnes, L.G., 2000, Identificación del delfín fósil del Mioceno Medio, Liolithax kernensis Kellogg 1931. Programa y Resúmenes, XXV Reunión Internacional para el Estudio de los Mamíferos Marinos, Sociedad Mexicana de Mastozoología Marina: La Paz, Baja California Sur, México, 7-11.

Fordyce, R.E., Barnes, L.G., 1994, The evolutionary history of whales and dolphins, en Wetherill, G.W. (ed.), 1994 Annual Review of Earth and Planetary Sciences, Volume 22: Annual Reviews, Inc., Palo Alto, California, 419 - 455.

Gastil, R.G., Phillips, R.P., Allison, E.C., 1975, Reconnaissance geology of the State of Baja California: Geological Society of America Mem, 140, 1-170.

González-Barba, G., 1990, Descripción de la fauna selácea del Miembro Los Indios de la Formación Playa Rosarito, Baja California, México: Universidad Autónoma de Baja California, Facultad de Ciencias marinas, tesis de licenciatura, $89 \mathrm{p}$.

Hawkins, J.W., 1970, Petrology and possible tectonic significance of Late Cenozoic volcanic rocks, southern California and Baja California: Geological Society of American Bulletin, 81, 3323-3338.

Ichishima H., 1995, A new kentriodontid dolphin (Cetacea; Kentriodontidae) from the Middle Miocene Takinoue Formation, Hokkaido, Japan: Island Arc, 3, 473-485.

Ichishima, H., Barnes, L.G., Fordyce, R.E., Kimura, M., Bohaska, D.J., 1995, A review of kentriodontine dolphins (Cetacea; Delphinoidea; Kentriodontidae): systematics and biogeography: Island Arc, 3(4), 486-492

Kazár, E., 2005, A new kentriodontid (Cetacea: Delphinoidea) from the Middle Miocene of Hungary: Fossil Record, 8(1), 53-78.

Kellogg, A.R., 1927, Kentriodon pernix, a Miocene porpoise from Maryland: Proceedings of the United States National Museum 69(19), 1-55, pls. 1-14.

Kellogg, A.R., 1931, Pelagic mammals from the Temblor Formation of the Kern River region, California: Proceedings of the California Academy of Sciences, Series 4, 19(12), 217-397.

Kellogg, A.R., 1955, Three Miocene porpoises from the Calvert Cliffs, Maryland: Proceedings of the United States National Museum, 105, 101-154, pls. 1-21.

Lambert, O., Estevens, M., Smith, R., 2005, A new kentriodontinae dolphin from the middle Miocene of Portugal: Acta Paleontologica Polonica. 50(2), 239-248

Lim, J.D., 2005, The first dolphin from the Miocene of Korea: Current Science, 89(6), 939-940.

Linnaeus, C., 1758, Systema naturae per regna tria naturae, secundum classes, ordines, genera, species, cum characteribus, differentiis synonymis, locis. Ed. 10, Tomus 1. L: Salvii, Stckholm, Sweden, $823 \mathrm{p}$.

Minch, J.A., 1967, Stratigraphy and structure of the Tijuana-Rosarito Beach area, northwestern Baja California, Mexico: Bulletin of the Geological Society of America, 78, 1155-1178.

Minch, J.A., Ashby, J.R., Deméré, T.A., Kuper, H.T., 1984, Correlation and depositional environments of the Middle Miocene Rosarito Beach Formation of northwestern Baja California, México, en Minch, J.A., Ashby, J.R. (eds.), Miocene and Cretaceous Depositional Environments, Northwestern Baja California, México: American Association of Petroleum Geologists, Pacific Section, 54, 33-46. 
Minch, J.A., Schulte, C.K., Hoffman, G., 1970, A Middle Miocene age for the Rosarito Beach Formation in northwestern Baja California, Mexico: Bulletin of the Geological Society of America, 81, 31493154 .

Montagu, G., 1821, Description of a species of Delphinus, which appears to be new: Memoirs of the Wernerian Natural History Society, 3, 75-82.

Perrin, W.F., 1975, Variation of spotted and spinner porpoise (gens Stenella) in the Eastern Tropical Pacific and Hawaii: Bulletin of the Scripps Institution of Oceanography, University of California Press, 21, 1-206.

Ralls, K., Mesnick, S.L., 2002, Sexual Dimorphism, en Perrin, W.F., Würsig, B., Thewissen, J.G.M. (eds.), Encyclopedia of Marine Mammals: Academic Press, 1071-1078.

Rensberger, J.M., 1969, A new Inid Cetacean from the Miocene of California: University of California Publications in Geological Sciences. 82, 1-43.

Rommel, S.A., Reynolds, J.E., 2002, Skeletal Anatomy, en Perrin, W.F., Thewissen, H., Wursig, B. (eds.), Encyclopedia of Marine Mammals: Academic Press, 1089-1103.

Salinas-Márquez, F.M., 2011, Kentriodonidae (Cetacea: Delphnoidea) de la fauna La Misión, Miceno Medio: Universidad Autónoma de Baja California, Facultad de Ciencias Marinas, Tesis de Maestría, 136 pp.
Shaffer, H.B., Meylan, P., McKnight, M.L., 1997, Tests of Turtle Phylogeny: Molecular morphological and paleontological approaches: Systematic Biology, 46(2), 235-268.

Slijper, E.J., 1936, Die Cetacean. Vergleichend-anatomisch and systematisch: Capita Zoologica, 6, i-xv, 1-590.

Swofford, D.L., 2002, PAUP*. Phylogenetic Analysis Using Parsimony (*and Other Methods). Version 4. Sinauer Associates, Sunderland, Massachusetts.

Wiley, E.O., Siegel-Causey, D., Brooks D.R., Funk, V.A., 1991, The complete cladist. A Primer of Phylogenetic Procedures: The University of Kanssas Museum of Natural History. Special Publication, 19, 158 .

Manuscrito recibido: Octubre 10, 2012.

Manuscrito corregido recibido: Enero 5. 2013.

Manuscrito aceptado: Febrero 7, 2013. 